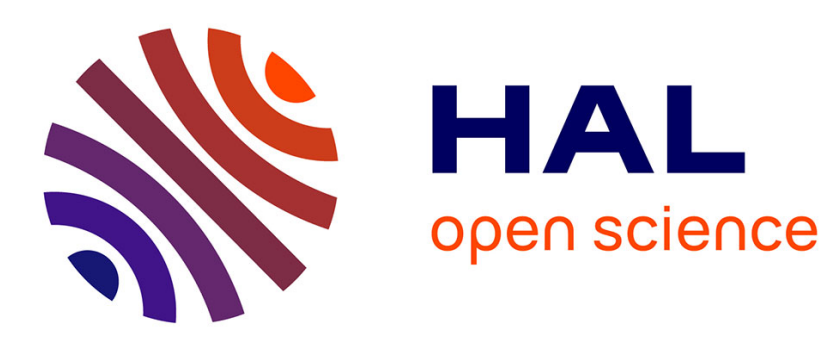

\title{
A Domain Decomposition Method for the Polarizable Continuum Model based on the Solvent Excluded Surface
}

\author{
Chaoyu Quan, Benjamin Stamm, Yvon Maday
}

\section{To cite this version:}

Chaoyu Quan, Benjamin Stamm, Yvon Maday. A Domain Decomposition Method for the Polarizable Continuum Model based on the Solvent Excluded Surface. 2018. hal-01489262v2

\section{HAL Id: hal-01489262 \\ https://hal.science/hal-01489262v2}

Preprint submitted on 26 Feb 2018

HAL is a multi-disciplinary open access archive for the deposit and dissemination of scientific research documents, whether they are published or not. The documents may come from teaching and research institutions in France or abroad, or from public or private research centers.
L'archive ouverte pluridisciplinaire HAL, est destinée au dépôt et à la diffusion de documents scientifiques de niveau recherche, publiés ou non, émanant des établissements d'enseignement et de recherche français ou étrangers, des laboratoires publics ou privés. 


\title{
A Domain Decomposition Method for the Polarizable Continuum Model based on the Solvent Excluded Surface*
}

\author{
Chaoyu Quan ${ }^{1}$, Benjamin Stamm², and Yvon Maday ${ }^{1,4,5}$ \\ ${ }^{1}$ Sorbonne Universités, UPMC Univ Paris 06, UMR 7598, Laboratoire Jacques-Louis Lions, F-75005, \\ Paris, France (quan@ann.jussieu.fr, maday@ann.jussieu.fr) \\ ${ }^{2}$ Center for Computational Engineering Science, RWTH Aachen University, Aachen, Germany \\ (best@mathcces.rwth-aachen.de) \\ ${ }^{4}$ Institut Universitaire de France, 75005, Paris, France \\ ${ }^{5}$ Division of Applied Mathematics, Brown University, 182 George St, Providence, RI 02912, USA
}

\begin{abstract}
In this article, an efficient solver for the polarizable continuum model in quantum chemistry is developed which takes the solvent excluded surface (the smooth molecular surface) as the solute-solvent boundary. This model requires to solve a generalized Poisson (GP) equation defined in $\mathbb{R}^{3}$ with a space-dependent dielectric permittivity function. First, the original GPequation is transformed into a system of two coupled equations defined in a bounded domain. Then, this domain is decomposed into overlapping balls and the Schwarz domain decomposition method is used. This method involves a direct Laplace solver and an efficient GP-solver to solve the local sub-equations in balls. For each solver, the spherical harmonics are used as basis functions in the angular direction of the spherical coordinate system. A series of numerical experiments are presented to test the performance of this method.
\end{abstract}

Keywords: Domain decomposition method; Polarizable continuum model; Solvent excluded surface

\section{Introduction}

Many physical and chemical phenomena of interest in chemistry and biology take place in the liquid phase, and the solvent effects consequently play a crucial role in these processes. The typical situation is that a solute molecule ( biological molecule for example) is embedded in the solvent. In this case, there are commonly two approaches to describing the solvent effects on the solute. The first one is to use an explicit solvation model, in which the simulated chemical system is composed of the solute molecule and a large number of explicit solvent molecules. The second one is to use an implicit solvation model, in which the solute molecule is embedded in a cavity surrounded by a continuous medium representing the solvent, i.e., the average response of the solvent molecules in

\footnotetext{
${ }^{*}$ Accepted by Mathematical Models and Methods in Applied Sciences on January 24, 2018.
} 
the sense of statistical mechanics. Note that the cavity here is not physical but is artificially defined in an ad hoc way.

The implicit solvation models commonly take into account the polarization effects of the solvent and involve elliptic operators from the mathematical point of view. Despite the fact that these models are widely used in the chemistry community, little interaction with applied mathematics can be observed. There are still many open problems, in particular on the suitable definition of the cavity, the nature of the continuum media, the sense in which it is a proper approximation, and also that, the solutions to partial differential equations (PDEs) need to be approximated.

In this article, we focus on developing an efficient solver for a popular implicit solvation model, the polarizable continuum model (PCM), in which the solvent is represented as a polarizable continuum (see [9, 48, 37] for an overview).

Within this article, the ESU-CGS (electrostatic units, centimetre-gram-second) system of units [20, Appendix $\mathrm{C}]$ is used for all equations.

\subsection{Problem statement}

Once the cavity surrounding the solute molecule is defined, the electrostatic potential $\psi$ in an implicit solvation model is described by the following partial different equation (PDE)

$$
-\nabla \cdot \varepsilon(\mathbf{x}) \nabla \psi(\mathbf{x})=4 \pi \rho(\mathbf{x}), \quad \text { in } \mathbb{R}^{3}
$$

where it is assumed that $\psi(\mathbf{x}) \sim \mathcal{O}\left(\frac{1}{|\mathbf{x}|}\right)$ as $|\mathbf{x}| \rightarrow \infty, \varepsilon(\mathbf{x})$ represents the space-dependent dielectric permittivity parameter and $\rho(\mathbf{x})$ represents the charge distribution of the solvation system.

Based on Eq. (1.1), one can derive two different models, respectively for the non-ionic solvent and the ionic solvent. First, one can obtain a generalized Poisson (GP) equation if $\rho$ is independent on $\psi$ as for the non-ionic solvent and second, a Poisson-Boltzmann equation [16] is obtained if the solvent contains ions whose movement is accounted for by Boltzmann statistics. Since the end of 1980s, many codes have been proposed for solving the Poisson-Boltzmann (or Poisson) equation for example using the boundary element method [7, 35], the finite element method [2, 24] or the finite difference method including UHBD [15, DelPhi [30, APBS [3, 17, 27] and the other work [18]. In particular, the APBS software is popular and widely used which can calculate the biomolecular electrostatics for large molecules. In the case of ionic solvent, the electrostatic potential decays exponentially with respect to the distance from the solute-solvent interface, which allows researchers to develop methods on bounded domains. However, in the case of non-ionic solvent, the electrostatic potential only decays as $\frac{1}{|\mathbf{x}|}$ due to the absence of ionic screening, which might require to take into account an integral representation of the potential on the entire unbounded domain for better accuracy. In this article, we focus on the case of non-ionic solvent and aim to solve the GP equation 1.1. for the PCM without ions.

In a classical PCM with solute cavity $\Omega$, the dielectric permittivity $\varepsilon(\mathbf{x})$ is defined as follows (see [11])

$$
\varepsilon(\mathbf{x})= \begin{cases}1 & \mathbf{x} \in \Omega \\ \varepsilon_{\mathrm{S}} & \mathbf{x} \in \Omega^{\mathrm{c}}:=\mathbb{R}^{3} \backslash \bar{\Omega}\end{cases}
$$

where $\varepsilon_{\mathrm{S}}$ is the solvent dielectric constant and $\varepsilon(\mathbf{x})$ has a jump on the solute-solvent boundary $\Gamma:=\partial \Omega$. Since the solvent is modeled as a polarizable continuum without ions, $\rho(\mathbf{x})$ is a function supported in the solute cavity. Physically speaking, this corresponds to the situation where the solute molecule is embedded in a homogeneous solvent of dielectric constant $\varepsilon_{\mathrm{s}}$. 
A reduced version of PCM is the conductor-like screening model (COSMO) 28 where the solvent is idealized as a conductor-like continuum, that is to say, $\varepsilon_{\mathrm{s}}=\infty$. In this case, the original equation (1.1) is simply a Laplace equation with homogeneous Dirichlet boundary condition

$$
\left\{\begin{aligned}
-\Delta \psi(\mathbf{x}) & =4 \pi \rho(\mathbf{x}) & & \text { in } \Omega, \\
\psi(\mathbf{x}) & =0 & & \text { on } \Gamma .
\end{aligned}\right.
$$

This reduced model is usually employed to approximate the PCM when the solvent dielectric constant $\varepsilon_{\mathrm{S}}$ is relatively large, for example, the (relative) dielectric constant of water is $\varepsilon_{\mathrm{S}}=78.4$ at room temperature $\left(25^{\circ} \mathrm{C}\right)$. To solve the COSMO, the finite element method or the finite difference method can be used. However, the computational cost is too large to obtain a high degree of accuracy for a large realistic molecule. In particular, meshing the solute cavity of a complicated molecule is already costly. In the past several years, a Schwarz domain decomposition method for solving the COSMO [43, 46] (called the ddCSOMO) has been developed, for the solute cavity constituted by a union of balls [10, 33, 31. This method has attracted much attention due to its impressive efficiency, that is, it performs about two orders of magnitude faster than the other equivalent methods [31]. Further, the convergence of the ddCOSMO has been analyzed recently in [13, where the contraction property is obtained in a simplified setting.

Later on, a similar Schwarz domain decomposition method to solve Eqs $\sqrt{1.1}-\sqrt{1.2}$ was proposed [47 (called the ddPCM), which is based on the integral equation formalism of the PCM [11]. The basic idea of the ddPCM (resp. the ddCOSMO) is to decompose the solute cavity into a union of balls and to solve each sub-equation in a ball, which avoids meshing the cavity. These two methods are limited to a domain constituted by the union of balls, such as the van der Waals (VdW) cavity and the solvent accessible surface (SAS) cavity [14, see the upcoming Section 2 where we will introduce those notions.

Due to the fast resolution of the COSMO and the PCM (see also website [32]), one starts to raise the question of a better definition of the solute cavity $\Omega$ in order to improve the methodology. The solvent excluded surface (SES, see Figure 22 cavity [14, 34, 41] seems to be a more suitable choice, because it has a stronger physical meaning that this cavity represents the region where the solvent molecules (represented by idealized spheres) can not touch. It has been confirmed in some chemical calculation that using the SES-cavity $\Omega_{\text {ses }}$ can yield more accurate results, such as in [44, 39, 40]. In fact, an SES-based solver for the implicit solvation model has been developed in [22, 8, using the integral equation of the PCM and an efficient mesh generator on the SES. In this article, we take $\Omega_{\text {ses }}$ as the solute cavity, i.e., $\Omega=\Omega_{\text {ses }}$. One should note that the SES has a more complicated geometry than the VdW surface and the SAS.

Further, there have been some attempts to introduce a continuous dielectric permittivity near the solute-solvent boundary [26, 19, 36, 5]. Mathematically speaking, this means that $\varepsilon(\mathbf{x})$ is also space-dependent in the solvent region:

$$
\varepsilon(\mathbf{x})= \begin{cases}1 & \mathbf{x} \in \Omega, \\ \varepsilon_{\mathrm{s}}(\mathbf{x}) & \mathbf{x} \in \Omega^{\mathrm{c}} .\end{cases}
$$

Due to the presence of the solute, the solvent density is not constant (especially for large biomolecules) near the solute-solvent boundary and therefore, the solvent dielectric permittivity is not constant. But at positions far from the solute molecule, the solvent dielectric permittivity $\varepsilon_{\mathbf{s}}(\mathbf{x})$ should be approximately the (bulk) solvent dielectric constant $\varepsilon_{\mathrm{s}}$, since the solvent molecules are influenced little by the solute molecule. Denoting by $\Omega_{\infty}$ the region far from the solute molecule, $\varepsilon_{\mathbf{s}}(\mathbf{x})$ then 


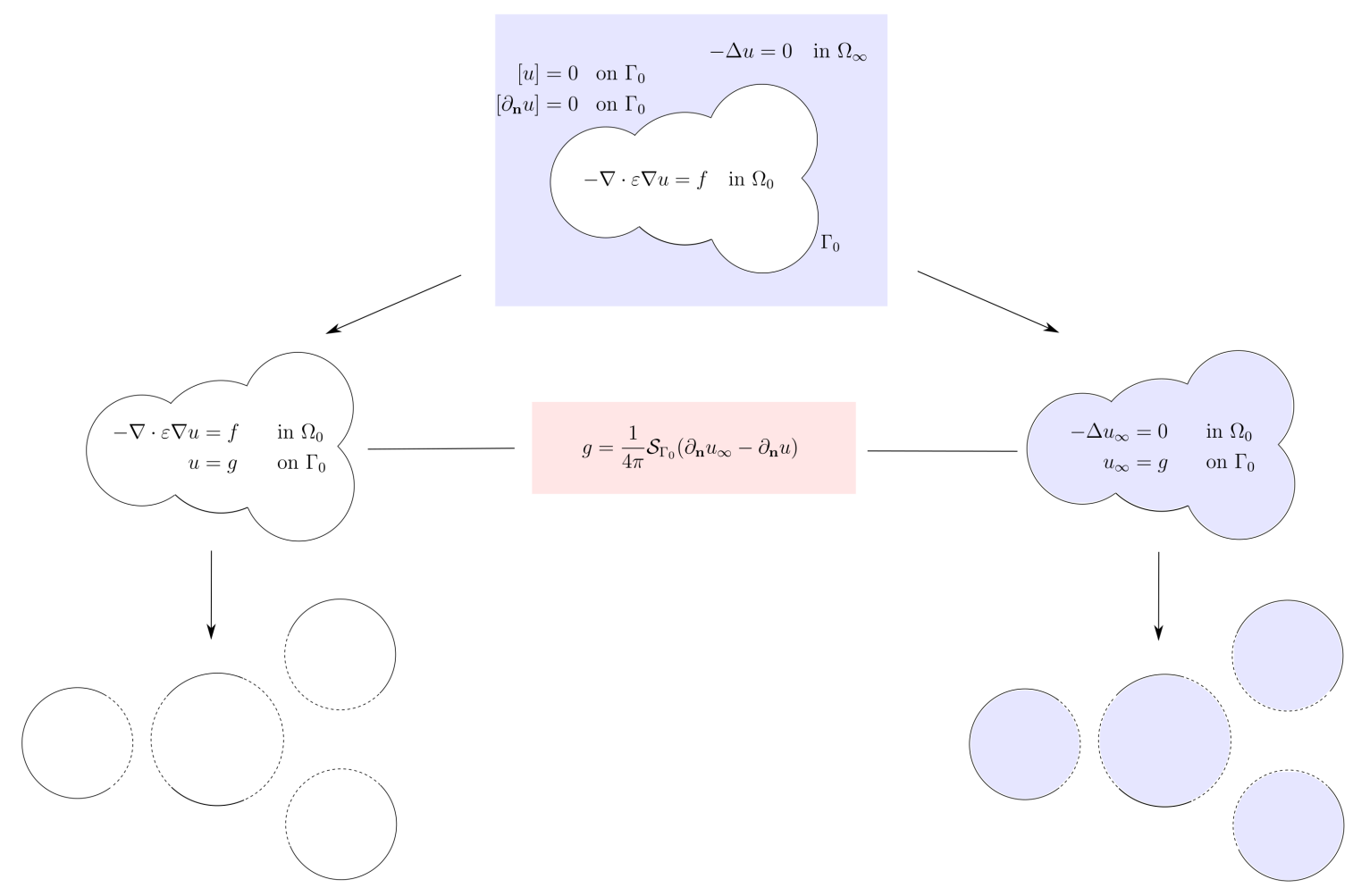

Figure 1: Schematic diagram of the domain decomposition method.

satisfies

$$
\varepsilon_{\mathrm{s}}(\mathbf{x})=\varepsilon_{\mathrm{s}}, \quad \text { in } \Omega_{\infty},
$$

which yields that $\psi$ of Eq. (1.1) is harmonic in $\Omega_{\infty}$, i.e. $-\Delta \psi=0$ in $\Omega_{\infty}$.

In summary, we want to solve Eq. (1.1) with a space-dependent parameter $\varepsilon(\mathbf{x})$ in the form of (1.4) - 1.5 where $\Omega$ is taken as $\Omega_{\text {ses }}$.

\subsection{Contribution}

The contribution of this article is two-fold. On the one hand, we construct a continuous dielectric permittivity function for the SES-based PCM. On the other hand, we introduce a Schwarz domain decomposition method to solve the established model. This method consists of transforming the original equation into two coupled (by a non-local condition) equations defined on a bounded domain, decomposing the bounded domain into balls and solving only local equations in balls by a spectral method.

The idea of this domain decomposition method is illustrated briefly in Figure 1. We consider 
the following problem in a general form

$$
\left\{\begin{aligned}
-\nabla \cdot \varepsilon \nabla u=f & \text { in } \Omega_{0}:=\Omega_{\infty}^{\mathrm{c}}, \\
-\Delta u=0 & \text { in } \Omega_{\infty} \\
{[u]=0 } & \text { on } \Gamma_{0}:=\partial \Omega_{0}, \\
{\left[\partial_{\mathbf{n}} u\right]=0 } & \text { on } \Gamma_{0},
\end{aligned}\right.
$$

where $[u]$ and $\left[\partial_{\mathbf{n}} u\right]$ denote the jump of $u$ and its normal derivative on $\Gamma_{0}, \varepsilon$ is a continuous function (in particular across $\Gamma_{0}$ ). The above equation can be transformed into two coupled equations defined on $\Omega_{0}$ as follows

$$
\left\{\begin{array} { r l } 
{ - \nabla \cdot \varepsilon \nabla u = f } & { \text { in } \Omega _ { 0 } , } \\
{ u = g } & { \text { on } \Gamma _ { 0 } , }
\end{array} \quad \text { and } \quad \left\{\begin{array}{rl}
-\Delta u_{\infty}=0 & \text { in } \Omega_{0}, \\
u_{\infty}=g & \text { on } \Gamma_{0},
\end{array}\right.\right.
$$

where a coupling condition arises through the auxiliary function $g$ defined as

$$
g=\frac{1}{4 \pi} \mathcal{S}_{\Gamma_{0}}\left(\partial_{\mathbf{n}} u_{\infty}-\partial_{\mathbf{n}} u\right), \quad \text { on } \Gamma_{0} .
$$

The operator $\mathcal{S}_{\Gamma_{0}}: H^{-\frac{1}{2}}\left(\Gamma_{0}\right) \rightarrow H^{\frac{1}{2}}\left(\Gamma_{0}\right)$ is the single-layer operator on $\Gamma_{0}$, which maps the function $\partial_{\mathbf{n}} u_{\infty}-\partial_{\mathbf{n}} u$ to another function in $H^{\frac{1}{2}}\left(\Gamma_{0}\right)$, see Section 4.2 for the definition of $\mathcal{S}_{\Gamma_{0}}$. Here, $H^{-\frac{1}{2}}\left(\Gamma_{0}\right)$ and $H^{\frac{1}{2}}\left(\Gamma_{0}\right)$ denote the usual Sobolev spaces of order $\pm \frac{1}{2}$ on the surface $\Gamma_{0}$, see [1]. The bounded domain $\Omega_{0}$ is taken as a union of balls, derived from the geometric data structures of the solute molecule. Thus, we can use the Schwarz domain decomposition method and only solve local problems in balls.

Ultimately, a Laplace solver and a GP solver are developed respectively to solve the Laplace equation and the GP equation in a ball. For each solver, we propose an efficient spectral method within each ball, using the spherical harmonics as basis functions in the angular direction. It is important to note that this algorithm does not require any mesh, but only involves problems in balls that are coupled to each other.

\subsection{Outline}

In Section 2, we first introduce different solute-solvent boundaries including the VdW surface, the SAS and the SES, which are fundamental and classical concepts of the implicit solvation models, which however are mostly unknown to the applied mathematics community. In Section 3, we construct a continuous dielectric permittivity function $\varepsilon(\mathbf{x})$ of PCM, ensuring that the SES-cavity always has the dielectric constant of vacuum. Then, in Section 4 we present the electrostatic problem of the PCM, its equivalent transformation, and a global iterative strategy for solving it. In Section 5, we introduce the scheme of the domain decomposition method for solving the associated partial differential equations in the global strategy. This requires to develop a Laplace solver and a GP-solver in the ball, which are presented in Section 6. After that, in Section 7, we give a series of numerical experiments on the performance of the proposed method. In the last section, we draw some conclusions.

\section{Solute-solvent boundary}

An important ingredient of an implicit solvation model is the solute-solvent boundary, that is, the interface between the solute and the solvent. 


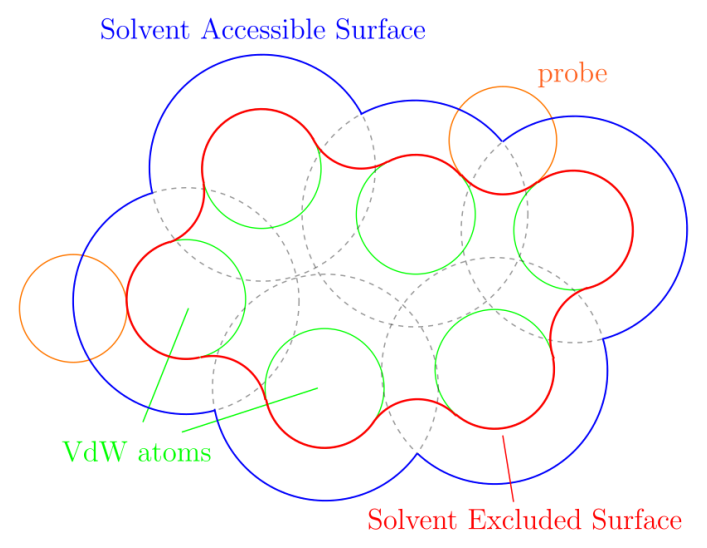

Figure 2: 2D schematic diagram of the VdW surface (green), the SAS (blue) and the SES (red).

Indeed, the choice of a proper solute cavity is important as pointed out in [49, Section II. C.]: The shape and size of the cavity are critical factors in the elaboration of a method. An ideal cavity should reproduce the shape of the solute, with the inclusion of the whole charge distribution and with the exclusion of empty spaces which can be filled by the solvent continuous distribution. If the cavity is too large, the solvation effects are damped; if it is too small, serious errors may arise in the evaluation of the interaction energy for the portions (atoms or bonds) near the solute-solvent boundary. One simple choice of the solute-solvent boundary is the VdW surface which is the topological boundary of the union of solute's VdW-atoms with radii experimentally fitted. Another choice is the SAS denoted by $\Gamma_{\mathrm{sas}}$, which is defined by tracing the center of an idealized (spherical) solvent probe (representing a solvent molecule) when rolling over the solute molecule. The region enclosed by the SAS is called the SAS-cavity, denoted by $\Omega_{\text {sas }}$. See Figure 2 for a 2D schematic diagram of different molecular surfaces.

The problem is that the VdW-cavity and the SAS-cavity are topologically not the correct answers to the cavity problem as they describe poorly the region where the solvent can touch. However, they are topologically simple and therefore attractive for numerical computations. Another solutesolvent boundary is the SES denoted by $\Gamma_{\text {ses }}$, which represents the boundary of the region where the probe has no access due to the presence of the solute. The region enclosed by the SES is the SES-cavity, denoted by $\Omega_{\text {ses }}$. See Figure 3 for a graphical illustration of the SAS and the SES of caffeine derived from our meshing algorithm 41, 42. In fact, any point on the SES has a constant distance to the SAS, equal to the solvent probe radius. From the geometrical point of view, the SES is smoother but also more complicated than the SAS.

To set the notations, we suppose that the solute molecule is composed of $M$ atoms and the $j$ th atom has center $\mathbf{c}_{j}$ and $\mathrm{VdW}$ radius $r_{j}$. The solvent probe radius is denoted by $r_{\mathrm{p}}$. Furthermore, for each atom, we define an "enlarged" ball $\Omega_{j}$ with center $\mathbf{c}_{j}$ and radius $R_{j}=r_{j}+r_{\mathrm{p}}+r_{0}$, where $r_{0}$ is a nonnegative constant. It is assumed that the solvent dielectric permittivity is constant outside the union of these enlarged balls. This is reasonable because the solvent density at positions far from the solute molecule (bulk) is approximately the same and therefore, the dielectric permittivity determined by the solvent density is almost the same [19, 5]. The SES-cavity is completely covered 

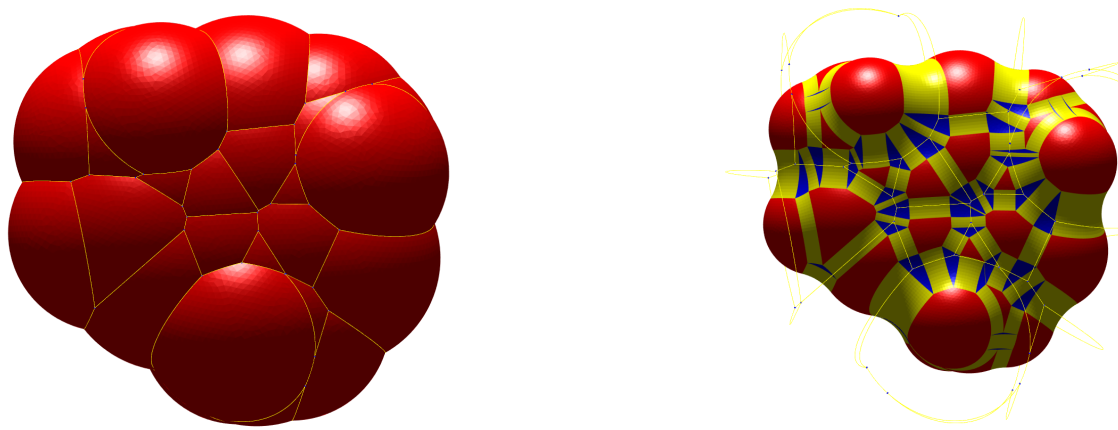

Figure 3: The SAS (left, $\Gamma_{\text {sas }}$ ) and the SES (right, $\Gamma_{\text {ses }}$ ) of caffeine. The SES is composed of convex spherical patches (red), toroidal patches (yellow) and concave spherical patches (blue).

by the union $\Omega_{0}$ of enlarged balls, that is,

$$
\Omega_{\mathrm{ses}} \subset \Omega_{0}:=\bigcup_{j=1}^{M} \Omega_{j}, \quad \text { with } \quad \Omega_{j}:=B_{R_{j}}\left(\mathbf{c}_{j}\right) .
$$

Here, we have specified the notation $\Omega_{0}$ mentioned in the introduction.

Denote the signed distance function to the SAS by $f_{\text {sas }}$ (negative inside the SAS and positive outside the SAS). We then have a mathematical characterization of the two cavities:

$$
\Omega_{\mathrm{ses}}=\left\{\mathbf{x} \in \mathbb{R}^{3}: f_{\mathrm{sas}}(\mathbf{x}) \leq-r_{\mathrm{p}}\right\} \quad \text { and } \quad \Omega_{0}=\left\{\mathbf{x} \in \mathbb{R}^{3}: f_{\mathrm{sas}}(\mathbf{x}) \leq r_{0}\right\} .
$$

Also, we have a characterization of their boundary surfaces

$$
\Gamma_{\mathrm{ses}}=f_{\mathrm{sas}}^{-1}\left(-r_{\mathrm{p}}\right) \quad \text { and } \quad \Gamma_{0}=f_{\mathrm{sas}}^{-1}\left(r_{0}\right) .
$$

An efficient method was proposed in 41 to compute $f_{\text {sas }}$ analytically, which is based on finding a closest point on the SAS to an arbitrary point. This allows us to construct a continuous permittivity function based on the value of $f_{\text {sas }}$.

\section{Dielectric permittivity function}

In this section, we construct a SES-based dielectric permittivity function $\varepsilon(\mathbf{x})$ associated with $f_{\text {sas }}$ as mentioned above.

In the following papers about implicit solvation models [26, 19, 36, 5] (see [48, Section 4.5] for more references), the motivation for taking into account continuous dielectric permittivity functions comes from the fact that the solvent density varies quite importantly around the solute molecule, especially for large biomolecules. As a consequence of this variation of the solvent density itself between near the solute-solvent boundary and at a faraway distance, there is a modification of the value of dielectric permittivity.

Taking the SES as the solute-solvent boundary implies that the dielectric permittivity in the SES-cavity is always 1, i.e., the dielectric constant of vacuum. As assumed in the previous section, the dielectric permittivity outside the enlarged cavity $\Omega_{0}$ is the solvent dielectric constant $\varepsilon_{\mathrm{s}}$. 


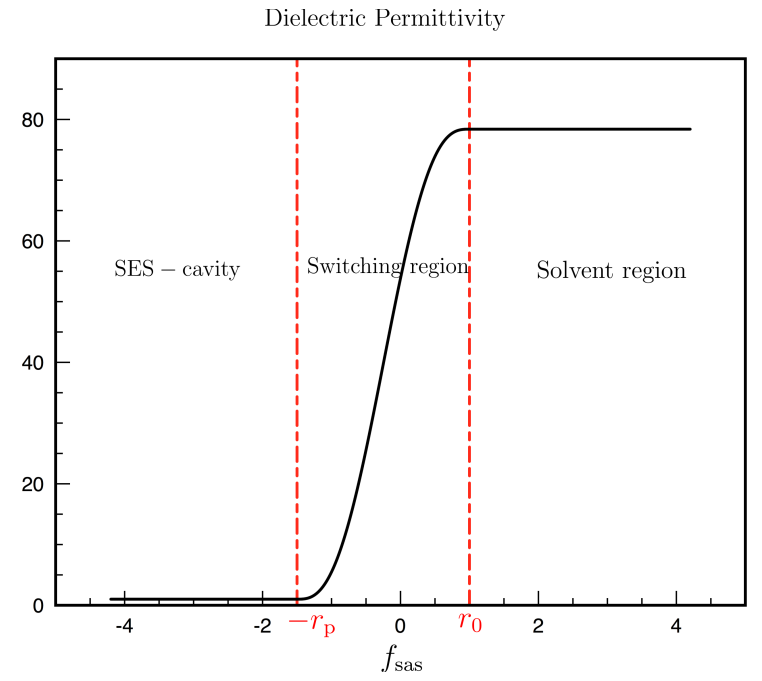

Figure 4: Schematic diagram of the dielectric permittivity function $\varepsilon(\mathbf{x})$ with respect to $f_{\text {sas }}$. The intermediate layer $\mathcal{L}$ (switching region) is bounded by two dashed lines (red), i.e., the region where $-r_{\mathrm{p}} \leq f_{\mathrm{sas}} \leq r_{0}$.

The remaining work is to determine $\varepsilon(\mathbf{x})$ in the intermediate layer (the switching region) $\mathcal{L}:=$ $\Omega_{0} \backslash \Omega_{\text {ses }}$. To construct a more physical permittivity function, we propose the following definition of $\varepsilon(\mathbf{x})$ depending on $f_{\text {sas }}$,

$$
\varepsilon(\mathbf{x})= \begin{cases}1 & \mathbf{x} \in \Omega_{\mathrm{ses}}, \\ \xi\left(\frac{f_{\mathrm{sas}}(\mathbf{x})+r_{\mathrm{p}}}{r_{\mathrm{p}}+r_{0}}\right) & \mathbf{x} \in \mathcal{L}, \\ \varepsilon_{\mathrm{s}} & \mathbf{x} \in \Omega_{\infty}:=\Omega_{0}^{\mathrm{c}},\end{cases}
$$

where $\xi$ is a continuous function defined on $[0,1]$, satisfying $\xi(0)=1, \xi(1)=\varepsilon_{\mathbf{s}} \cdot \varepsilon(\mathbf{x})$ can be seen as a distance-dependent function where the "distance" represents the signed distance to the SAS, see Figure 4 for a schematic diagram. To guarantee the smoothness of the dielectric permittivity on the interfaces $\Gamma_{\text {ses }}$ and $\Gamma_{0}$, one can impose more conditions to the function $\xi$, for example,

$$
\xi^{\prime}(0)=0, \quad \xi^{\prime}(1)=0, \quad \xi^{\prime \prime}(0)=0, \quad \xi^{\prime \prime}(1)=0 .
$$

Remark 3.1. We emphasize that the function $\xi$ can be chosen in many different manners satisfying various conditions. This characterizes the way the dielectric permittivity varies in the intermediate switching region.

The above SES-based PCM can be used to approximate the classical PCM with the discontinuous dielectric permittivity function (1.2). If one chooses a sequence of functions $\xi(t)$ approaching the discontinuous function

$$
\chi_{\mathrm{ses}}(t)= \begin{cases}1 & t<0, \\ \varepsilon_{\mathrm{s}} & t \geq 0,\end{cases}
$$


then the governing equations of the SES-based PCM approach the equations of the classical PCM with $\Omega=\Omega_{\text {ses. }}$. Similarly, if one chooses another sequence of functions $\xi(t)$ approaching the discontinuous function

$$
\chi_{\mathrm{sas}}(t)= \begin{cases}1 & t<1, \\ \varepsilon_{\mathrm{s}} & t \geq 1,\end{cases}
$$

then the governing equations of the SES-based PCM approach the equations of the classical PCM with $\Omega=\Omega_{\text {sas }}$.

\section{Problem formulation and global strategy}

In this section, we first introduce the electrostatic problem of the PCM and then present the techniques of transforming the original problem into two coupled problems defined on a bounded domain. After the transformation, we propose a global iterative strategy to solve the electrostatic problem.

\subsection{Problem formulation}

One crucial issue on the PCM is to compute the electrostatic contribution to the solvation energy, denoted by $E^{\mathrm{s}}$. This electrostatic contribution can be written as

$$
E^{\mathrm{S}}=\frac{1}{2} \int_{\mathbb{R}^{3}} \rho(\mathbf{x}) W(\mathbf{x}) \mathrm{d} \mathbf{x},
$$

where $W$ denotes the (solvent) reaction potential generated by the solute's charge density $\rho$ in presence of the polarizable continuum. The governing equations of the potential $W$ will be explained later in this section.

We assume that the electrostatic potential $\Phi$ generated by the charge distribution $\rho$ in vacuum is already known and $\rho$ is supported in $\Omega_{\text {ses }}$, i.e. $\operatorname{supp}(\rho) \subset \Omega_{\text {ses }}$, which is a standard assumption in the content of PCM. As a consequence, $\Phi$ satisfies the following PDE

$$
-\Delta \Phi(\mathbf{x})=4 \pi \rho(\mathbf{x}), \quad \text { in } \mathbb{R}^{3} .
$$

As in [10], one always takes a neutral solute molecule with a classical charge distribution for simplicity,

$$
\rho(\mathbf{x})=\sum_{j=1}^{M} q_{j} \delta_{\mathbf{c}_{j}}(\mathbf{x}), \quad \forall \mathbf{x} \in \mathbb{R}^{3},
$$

where $q_{j}$ represents the charge of the $j$ th atom and $\delta_{\mathbf{c}_{j}}$ is the Dirac function at the atomic center $\mathbf{c}_{j}$. Then, the electrostatic potential $\Phi$ generated by $\rho$ is derived

$$
\Phi(\mathbf{x})=\sum_{j=1}^{M} \frac{q_{j}}{\left|\mathbf{x}-\mathbf{c}_{j}\right|}, \quad \forall \mathbf{x} \in \mathbb{R}^{3} .
$$

The reaction potential $W:=\psi-\Phi$ represents the difference between the electrostatic potentials with and without the presence of solvent. For a SES-based PCM with $\varepsilon(\mathbf{x})$ given in (3.7), $W$ 

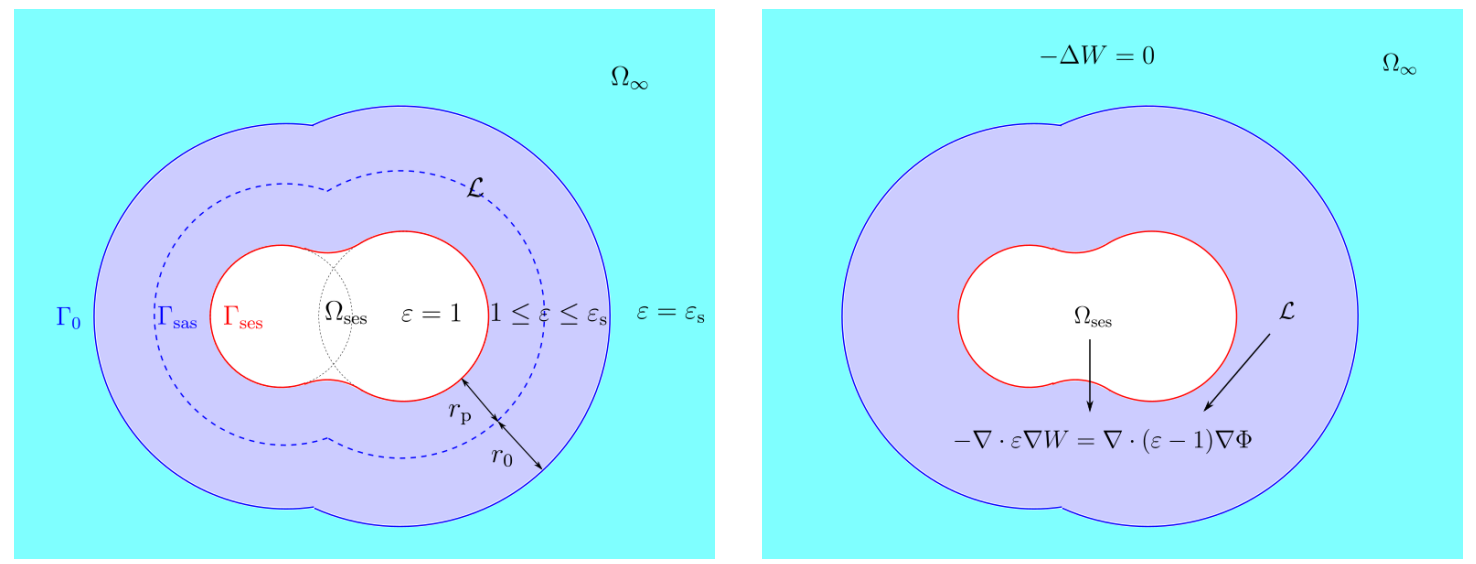

Figure 5: 2D schematic diagrams of some notations (left) and the PDEs of the PCM in different regions (right) for a system of two atoms.

satisfies consequently the following PDEs

$$
\left\{\begin{aligned}
-\Delta W=0 & \text { in } \Omega_{\mathrm{ses}}, \\
-\nabla \cdot \varepsilon \nabla(W+\Phi)=0 & \text { in } \mathcal{L}, \\
-\Delta W=0 & \text { in } \Omega_{\infty} .
\end{aligned}\right.
$$

Further, since $\varepsilon(\mathbf{x})$ is continuous across the interfaces $\Gamma_{\text {ses }}$ and $\Gamma_{0}$, the following classical jumpconditions are required

$$
\left\{\begin{aligned}
{[W] } & =0 & & \text { on } \Gamma_{\text {ses }} \text { and } \Gamma_{0}, \\
{\left[\partial_{\mathbf{n}} W\right] } & =0 & & \text { on } \Gamma_{\text {ses }} \text { and } \Gamma_{0},
\end{aligned}\right.
$$

where $[W]$ denotes the jump (inside minus outside) of the reaction potential $W$ on the boundary surfaces $\Gamma_{\text {ses }}$ and $\Gamma_{0},\left[\partial_{\mathbf{n}} W\right]$ denotes the jump of the normal derivative of the reaction potential, $\mathbf{n}$ denotes the unit normal vector on $\Gamma_{0}$ pointing outwards with respect to $\Omega_{0}, \partial_{\mathbf{n}} W$ denotes the normal derivative $\nabla W \cdot \mathbf{n}$.

\subsection{Equivalent transformation}

Since $\operatorname{supp}(\rho) \subset \Omega_{\text {ses }}$, we have $-\Delta \Phi=0$ in $\mathcal{L}$. Combining with $\varepsilon(\mathbf{x})=1$ in $\Omega_{\text {ses }}$, the above Eqs 4.15 - 4.16 can be recast as

$$
\left\{\begin{aligned}
-\nabla \cdot \varepsilon \nabla W & =\nabla \cdot(\varepsilon-1) \nabla \Phi & & \text { in } \Omega_{0}, \\
-\Delta W & =0 & & \text { in } \Omega_{\infty},
\end{aligned}\right.
$$

with

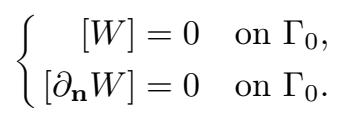

See Figure 5 for the schematic diagram of the PDEs in different regions. 
In the second equation of 4.17$)$, a single layer potential $\widetilde{\mathcal{S}}_{\Gamma_{0}}: H^{-\frac{1}{2}}\left(\Gamma_{0}\right) \rightarrow H^{1}\left(\mathbb{R}^{3}\right)$ can be used to represent the electrostatic potential $W$ restricted in $\Omega_{\infty}$ as follows

$$
\left.W\right|_{\Omega_{\infty}}(\mathbf{x})=\left(\widetilde{\mathcal{S}}_{\Gamma_{0}} \sigma\right)(\mathbf{x}):=\int_{\Gamma_{0}} \frac{\sigma\left(\mathbf{s}^{\prime}\right)}{\left|\mathbf{x}-\mathbf{s}^{\prime}\right|} \mathrm{d} \mathbf{s}^{\prime}, \quad \forall \mathbf{x} \in \Omega_{\infty},
$$

where $H^{-\frac{1}{2}}\left(\Gamma_{0}\right)$ and $H^{1}\left(\Gamma_{0}\right)$ denote the Sobolev spaces, $\sigma$ is some function in $H^{-\frac{1}{2}}\left(\Gamma_{0}\right)$. From the continuity of the single-layer potential across the interface, see for example [45, 21], we take the limit to $\Gamma_{0}$, so as to obtain the integral equation

$$
\left.W\right|_{\Gamma_{0}}(\mathbf{s})=\left(\mathcal{S}_{\Gamma_{0}} \sigma\right)(\mathbf{s}):=\int_{\Gamma_{0}} \frac{\sigma\left(\mathbf{s}^{\prime}\right)}{\left|\mathbf{s}-\mathbf{s}^{\prime}\right|} \mathrm{d} \mathbf{s}^{\prime}, \quad \forall \mathbf{s} \in \Gamma_{0},
$$

where $\mathcal{S}_{\Gamma_{0}}: H^{-\frac{1}{2}}\left(\Gamma_{0}\right) \rightarrow H^{\frac{1}{2}}\left(\Gamma_{0}\right)$ is the single-layer operator which is invertible (see also [45, 21]) and thus defines $\sigma \in H^{-\frac{1}{2}}\left(\Gamma_{0}\right)$. Further, we can artificially extend the potential in 4.19 to $\Omega_{0}$ as follows

$$
W_{\infty}(\mathbf{x}):=\int_{\Gamma_{0}} \frac{\sigma\left(\mathbf{s}^{\prime}\right)}{\left|\mathbf{x}-\mathbf{s}^{\prime}\right|} \mathrm{d} \mathbf{s}^{\prime}, \quad \forall \mathbf{x} \in \Omega_{0},
$$

satisfying

$$
-\Delta W_{\infty}=0, \quad \text { in } \Omega_{0}
$$

and

$$
W_{\infty}=\left.W\right|_{\Omega_{\infty}}, \quad \text { on } \Gamma_{0} .
$$

Recalling the relationship between the charge density $\sigma$ and the jump of the normal derivative of the potential on $\Gamma_{0}$ generated by $\sigma$ [47, we actually have

$$
\sigma=\frac{1}{4 \pi}\left(\partial_{\mathbf{n}} W_{\infty}-\left.\partial_{\mathbf{n}} W\right|_{\Omega_{\infty}}\right), \quad \text { on } \Gamma_{0} .
$$

Combining with $\left[\partial_{\mathbf{n}} W\right]=0$ on $\Gamma_{0}$ in 4.18 , i.e., $\left.\partial_{\mathbf{n}} W\right|_{\Omega_{0}}=\left.\partial_{\mathbf{n}} W\right|_{\Omega_{\infty}}$, we therefore obtain

$$
\sigma=\frac{1}{4 \pi}\left(\partial_{\mathbf{n}} W_{\infty}-\left.\partial_{\mathbf{n}} W\right|_{\Omega_{0}}\right), \quad \text { on } \Gamma_{0} .
$$

Also, since $[W]=0$ in 4.18$)$ and $W_{\infty}=\left.W\right|_{\Omega_{\infty}}$ on $\Omega_{0}$, we have $W_{\infty}=W$ on $\Gamma_{0}$.

Finally, let's summarize what we have in hand now. We have obtained two PDEs of $W$ and $W_{\infty}$ both defined on $\Omega_{0}$

$$
\left\{\begin{aligned}
-\nabla \cdot \varepsilon \nabla W & =\nabla \cdot(\varepsilon-1) \nabla \Phi & & \text { in } \Omega_{0} \\
-\Delta W_{\infty} & =0 & & \text { in } \Omega_{0},
\end{aligned}\right.
$$

and two boundary conditions coupling them

$$
\left\{\begin{aligned}
W & =W_{\infty} & & \text { on } \Gamma_{0}, \\
\sigma & =\frac{1}{4 \pi}\left(\partial_{\mathbf{n}} W_{\infty}-\partial_{\mathbf{n}} W\right) & & \text { on } \Gamma_{0},
\end{aligned}\right.
$$

where $\sigma$ is the density generating $W_{\infty}$ by 4.21). To compute the electrostatic contribution to the solvation energy, we need to compute $W$ which involves solving Eqs (4.26)-(4.27). Although we solve for a problem in an unbounded domain, we need to solve equations only on the bounded domain $\Omega_{0}$ which is a remarkable property. 


\subsection{Global strategy}

From the above formulation, we propose the following iterative procedure for solving Eqs 4.26 (4.27): Let $g^{0}$ be an initial guess for the Dirichlet condition $\left.W\right|_{\Gamma_{0}}=\left.W_{\infty}\right|_{\Gamma_{0}}$ on $\Gamma_{0}$ and set $k=1$.

[1] Solve the following Dirichlet boundary problem for $W^{k}$ :

$$
\left\{\begin{aligned}
-\nabla \cdot \varepsilon \nabla W^{k} & =\nabla \cdot(\varepsilon-1) \nabla \Phi & & \text { in } \Omega_{0}, \\
W^{k} & =g^{k-1} & & \text { on } \Gamma_{0},
\end{aligned}\right.
$$

and derive its Neumann boundary trace $\partial_{\mathbf{n}} W^{k}$ on $\Gamma_{0}$.

[2] Solve the following Dirichlet boundary problem for $W_{\infty}^{k}$ :

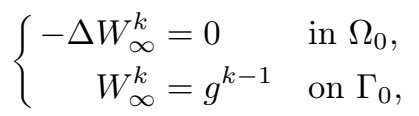

and derive similarly its Neumann boundary trace $\partial_{\mathbf{n}} W_{\infty}^{k}$ on $\Gamma_{0}$.

[3] Build the charge density $\sigma^{k}=\frac{1}{4 \pi}\left(\partial_{\mathbf{n}} W_{\infty}^{k}-\partial_{\mathbf{n}} W^{k}\right)$ and compute a new Dirichlet condition $g^{k}=\mathcal{S}_{\Gamma_{0}} \sigma^{k}$.

[4] Compute the electrostatic contribution $E_{k}^{\mathrm{s}}$ to the solvation energy following (4.11) based on $W^{k}$ at the $k$-th iteration, set $k \leftarrow k+1$, go back to Step [1] and repeat until the increment of electrostatic interaction $\left|E_{k}^{\mathrm{s}}-E_{k-1}^{\mathrm{s}}\right|$ becomes smaller than a given tolerance Tol $\ll 1$.

Remark 4.1. In order to provide a suitable initial guess of $g^{0}$, we consider the (unrealistic) scenario where the whole space $\mathbb{R}^{3}$ is covered by the solvent medium with the dielectric constant $\varepsilon_{\mathrm{s}}$. In consequence, the electrostatic potential $\psi$ is given by $\psi=\frac{1}{\varepsilon_{\mathrm{s}}} \Phi$ and the reaction potential is provided by $W=\psi-\Phi=\left(\frac{1}{\varepsilon_{\mathrm{s}}}-1\right) \Phi$. We therefore propose the following initial Dirichlet boundary function $g^{0}$ for the first iteration:

$$
g^{0}=\left(\frac{1}{\varepsilon_{\mathrm{s}}}-1\right) \Phi .
$$

Remark 4.2. This iterative procedure has the remarkable property that we solve a problem on an unbounded domain by a sequence of problems on bounded domains only. It can be seen as a domain decomposition method on the two non-overlapping domains $\Omega_{0}$ and $\Omega_{\infty}$ where only problems on the bounded domain $\Omega_{0}$ are solved. Similar property has been obtained for the integral equation formulation of implicit solvation models, see for example [12, 11, 29, 4].

In the next section, we propose to use the classical Schwarz domain decomposition method to solve the PDE (4.28) in Step [1] and the PDE (4.29) in Step [2] by introducing sub-iterations.

\section{Domain decomposition strategy}

The Schwarz domain decomposition method [43, 46] aims at solving partial differential equations defined on complex domains which can be decomposed as a union of overlapping and possibly simple subdomains. For each subdomain, the same equation is solved but with boundary conditions that 
depend on the global boundary condition on one hand and on the neighboring solutions on the other hand.

Recalling that we have a natural domain decomposition of $\Omega_{0}$ as follows

$$
\Omega_{0}=\bigcup_{j=1}^{M} \Omega_{j}, \quad \Omega_{j}=B_{R_{j}}\left(\mathbf{c}_{j}\right),
$$

the Schwarz domain decomposition method can be applied to solve the PDE (4.28). We replace the global equation 4.28 by the following coupled equations, each restricted to $\Omega_{j}$ :

$$
\left\{\begin{aligned}
-\nabla \cdot \varepsilon \nabla W_{j} & =\nabla \cdot(\varepsilon-1) \nabla \Phi & & \text { in } \Omega_{j} \\
W_{j} & =h_{j} & & \text { on } \Gamma_{j},
\end{aligned}\right.
$$

where $W_{j}=\left.W\right|_{\Omega_{j}}, \Gamma_{j}=\partial \Omega_{j}$ and

$$
h_{j}= \begin{cases}W_{j}^{\mathcal{N}} & \text { on } \Gamma_{j}^{\mathrm{i}}, \\ g & \text { on } \Gamma_{j}^{\mathrm{e}} .\end{cases}
$$

Here, we omit the superscript due to the (outer) iteration index $k . \Gamma_{j}^{\mathrm{e}}$ is the external part of $\Gamma_{j}$ not contained in any other ball $\Omega_{i}(i \neq j)$, i.e., $\Gamma_{j}^{\mathrm{e}}=\Gamma_{0} \cap \Gamma_{j}, \Gamma_{j}^{\mathrm{i}}$ is the internal part of $\Gamma_{j}$, i.e., $\Gamma_{j}^{\mathrm{i}}=\Omega_{0} \cap \Gamma_{j}$ (see Figure 6 for an illustration), and

$$
W_{j}^{\mathcal{N}}(\mathbf{s})=\frac{1}{|\mathcal{N}(j, \mathbf{s})|} \sum_{i \in \mathcal{N}(j, \mathbf{s})} W_{i}(\mathbf{s}), \quad \forall \mathbf{s} \in \Gamma_{j}^{\mathrm{i}},
$$

where $\mathcal{N}(j, \mathbf{s})$ represents the index set of all balls that overlap $\Omega_{j}$ at $\mathbf{s}$. In fact, for a fixed point $\mathbf{s} \in \Gamma_{j}^{\mathrm{i}}$, we enforce $W_{j}=W_{j}^{\mathcal{N}}(\mathbf{s})$.

In the next section, we will develop a GP-solver for solving the local problems (5.31). For each local problem defined on $\Omega_{j}$, this solver provides an approximate weak solution. Based on this solver, an iterative procedure can be applied to solve the coupled equations (5.31)-(5.32), such as the parallel and alternating Schwarz algorithms as presented in [10. The idea of the parallel Schwarz algorithm is to solve each local problem based on the boundary condition of the neighboring solutions derived from the previous iteration. During this iterative procedure, the computed value of $\left.W\right|_{\Gamma_{j}^{\mathrm{i}}}$ is updated step by step and converges to the exact value.

The parallel and alternating Schwarz algorithms might not be the most efficient way to solve this set of equations, but is well-suited to illustrate the idea of domain decomposition. In practice, a global problem (linear system after introducing a discretization) can be solved with GMRes for example.

Remark 5.1. Notice that the global strategy for computing the electrostatic contribution to the solvation energy in Section 4.3 is also an iterative process (it was indexed by $k$ ). To distinguish these two iterations, the global iteration in Section 4.3 is called the outer iteration and the iteration of solving the GP-equation (4.28) by iteratively solving the set of local problems (5.31) is called the inner iteration. Of course, for the sake of efficiency, these two iterations (inner and outer) should be intertwined. 


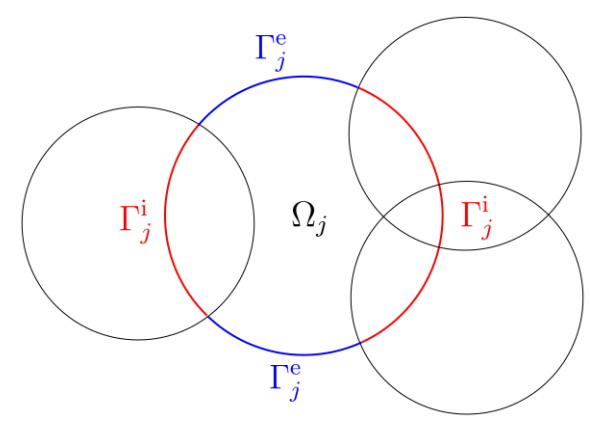

Figure 6: 2D schematic diagram of $\Gamma_{j}^{\mathrm{i}}$ (red) and $\Gamma_{j}^{\mathrm{e}}$ (blue) associated with $\Omega_{j}$.

As to solve the Laplace equation (4.29), we still use the domain decomposition method which is called the ddCOSMO method in this context and has been developed in [10]. In fact, Eq. 44.29] can been seen as a special case of Eq. 4.28 when $\varepsilon=1$. As a consequence, the same domain decomposition method as presented in $(5.31)-(5.32)$ can be used where each local problem (5.31) simplifies to a Laplace problem.

\section{Single-domain solvers}

\subsection{Laplace equation in a ball}

As seen above, it is required to solve a set of coupled Laplace equations, each of which is restricted to a ball as in the ddCOSMO. For the sake of completeness, we now introduce briefly the ideas. Without loss of generality, we consider the following Laplace equation with the Dirichlet boundary condition $\phi_{0}$ and defined in the unit ball with center $\mathbf{0}$ :

$$
\left\{\begin{aligned}
-\Delta u_{0}=0 & \text { in } B_{1}(\mathbf{0}) \\
u_{0}=\phi_{0} & \text { on } \partial B_{1}(\mathbf{0}),
\end{aligned}\right.
$$

where $B_{1}(\mathbf{0})$ represents the unit ball centered at the origin $\mathbf{0}$. As a consequence, the unique solution to 6.34 in $H^{1}\left(B_{1}(\mathbf{0})\right)$ can be written as

$$
u_{0}(r, \theta, \varphi)=\sum_{\ell=0}^{\infty} \sum_{m=-\ell}^{\ell}\left[\phi_{0}\right]_{\ell}^{m} r^{\ell} Y_{\ell}^{m}(\theta, \varphi), \quad 0 \leq r \leq 1,0 \leq \theta \leq \pi, 0 \leq \varphi<2 \pi,
$$

where $Y_{\ell}^{m}$ is the (orthonormal) spherical harmonic of degree $\ell$ and order $m$ defined on $\mathbb{S}^{2}$ and

$$
\left[\phi_{0}\right]_{\ell}^{m}=\int_{\mathbb{S}^{2}} \phi_{0}(\mathbf{s}) Y_{\ell}^{m}(\mathbf{s}) \mathrm{d} \mathbf{s}
$$

is the real coefficient of $u_{0}$ corresponding to the mode $Y_{\ell}^{m}$.

To compute 6.35 numerically, we first approximate $\left[\phi_{0}\right]_{\ell}^{m}$ using the Lebedev quadrature rule 23] defined by the integration points $\mathbf{s}_{n} \in \mathbb{S}^{2}$ and their weights $w_{n}^{\text {leb }}$ as follows

$$
\left[\widetilde{\phi}_{0}\right]_{\ell}^{m}=\sum_{n=1}^{N_{\text {leb }}} w_{n}^{\text {leb }} \phi_{0}\left(\mathbf{s}_{n}\right) Y_{\ell}^{m}\left(\mathbf{s}_{n}\right)
$$


where $N_{\text {leb }}$ represents the number of Lebedev points. Then, $u_{0}$ can be approximated by $\widetilde{u}_{0}$ in the discretization space spanned by a truncated basis of spherical harmonics $\left\{Y_{\ell}^{m}\right\}_{0 \leq \ell \leq \ell_{\max }},-\ell \leq m \leq \ell$, defined as

$$
\widetilde{u}_{0}(r, \theta, \varphi)=\sum_{\ell=0}^{\ell_{\max }} \sum_{m=-\ell}^{\ell}\left[\widetilde{\phi}_{0}\right]_{\ell}^{m} r^{\ell} Y_{\ell}^{m}(\theta, \varphi), \quad 0 \leq r \leq 1,0 \leq \theta \leq \pi, 0 \leq \varphi<2 \pi,
$$

where $\ell_{\max }$ denotes the maximum degree of spherical harmonics. This approximate solution $\widetilde{u}_{0}$ converges to the exact solution $u_{0}$ to Eq. 6.34 when $\ell_{\max } \rightarrow \infty$ and $N_{\text {leb }} \rightarrow \infty$. One should note however that there is no systematic manner to derive Lebedev points of arbitrary accuracy.

Remark 6.1. To compute the surface integral over the unit sphere, any other quadrature in the angular variables $\theta$ and $\varphi$ can be used. However, the Lebedev quadrature is very efficient, in the sense that fewer grid points are required to obtain similar accuracy, which has been pointed out in [6]. In addition, Lebedev grids yield exact numerical integration of spherical harmonics up to a certain degree (depending on the number of integration points) and satisfy some invariance properties of certain rotational groups.

\subsection{Generalized Poisson equation in a ball}

To solve the local equations (5.31), we need to develop a solver for the GP-equation defined on $\Omega_{j}$ with the Dirichlet boundary condition (5.32). Without loss of generality, we discuss how to solve the following GP-equation in the unit ball with center $\mathbf{0}$ in the general form of

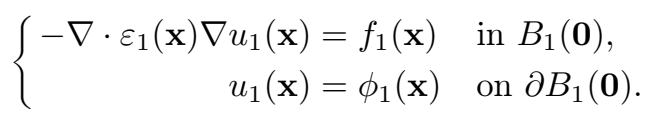

In fact, for any $j=1, \ldots, M$, we take $\varepsilon_{1}(\mathbf{x})=\varepsilon\left(\mathbf{c}_{j}+R_{j} \mathbf{x}\right), u_{1}(\mathbf{x})=W_{j}\left(\mathbf{c}_{j}+R_{j} \mathbf{x}\right), f_{1}(\mathbf{x})=$ $\nabla \cdot\left(\varepsilon_{1}(\mathbf{x})-1\right) \nabla \Phi\left(\mathbf{c}_{j}+R_{j} \mathbf{x}\right)$ and $\phi_{1}(\mathbf{x})=h_{j}\left(\mathbf{c}_{j}+R_{j} \mathbf{x}\right)$ where $\varepsilon, W_{j}, \Phi, h_{j}$ are as in Eqs (5.31)5.32 .

\subsubsection{Variational formulation}

From the discussion in Section 6.1 we know that there exists a unique harmonic function $\hat{u} \in$ $H^{1}\left(B_{1}(\mathbf{0})\right)$, s.t.

$$
\left\{\begin{aligned}
-\Delta \hat{u}_{1}=0 & \text { in } B_{1}(\mathbf{0}) \\
\hat{u}_{1}=\phi_{1} & \text { on } \partial B_{1}(\mathbf{0}),
\end{aligned}\right.
$$

and $\hat{u}_{1}$ can be efficiently approximated by Eq. 6.36). Let $v=u_{1}-\hat{u}_{1} \in H_{0}^{1}\left(B_{1}(\mathbf{0})\right)$ and in consequence, $v$ satisfies the following $\mathrm{PDE}$

$$
\left\{\begin{aligned}
-\nabla \cdot \varepsilon_{1} \nabla v=f & \text { in } B_{1}(\mathbf{0}) \\
v=0 & \text { on } \partial B_{1}(\mathbf{0}),
\end{aligned}\right.
$$

where $f(\mathbf{x})=\nabla \cdot\left(\varepsilon_{1}(\mathbf{x})-1\right) \nabla \Phi\left(\mathbf{c}_{j}+R_{j} \mathbf{x}\right)+\nabla \cdot \varepsilon_{1}(\mathbf{x}) \nabla \hat{u}_{1}(\mathbf{x})$.

Since the VdW-ball $B_{r_{j}}\left(\mathbf{c}_{j}\right) \subset \Omega_{j}$ and $\varepsilon \equiv 1$ holds in $B_{r_{j}}\left(\mathbf{c}_{j}\right)$, we know that $W_{j}$ defined in (5.31) is harmonic when restricted to the smaller ball $B_{r_{j}}\left(\mathbf{c}_{j}\right)$. As a consequence, $u_{1}$ of Eq. 6.37) and $v$ of Eq. 6.39) are both harmonic in $B_{\delta}(\mathbf{0})$, where $\delta$ is defined by

$$
\delta=\frac{r_{j}}{r_{j}+r_{0}+r_{\mathrm{p}}} \in(0,1) .
$$


Let $\mathcal{D}:=B_{1}(\mathbf{0}) \backslash \bar{B}_{\delta}(\mathbf{0})$ represent the region between $\partial B_{1}(\mathbf{0})$ and $\partial B_{\delta}(\mathbf{0})$ and define the subspace $H_{0, \delta}^{1}(\mathcal{D})$ of the Sobolev space $H^{1}(\mathcal{D})$ as follows

$$
H_{0, \delta}^{1}(\mathcal{D})=\left\{w \in H^{1}(\mathcal{D}):\left.w\right|_{\partial B_{1}(\mathbf{0})}=0\right\} .
$$

In order to find the weak solution restricted to $H_{0, \delta}^{1}(\mathcal{D})$, we can write a variational formulation as: find $v \in H_{0, \delta}^{1}(\mathcal{D})$, s.t. $\forall w \in H_{0, \delta}^{1}(\mathcal{D})$,

$$
\int_{\mathcal{D}} \varepsilon_{1} \nabla v \cdot \nabla w+\int_{\partial B_{\delta}(\mathbf{0})}(\mathcal{T} v) w=\int_{\mathcal{D}} f w
$$

where we use the fact that $\left.\varepsilon_{1}\right|_{\partial B_{\delta}(\mathbf{0})}=1$. The operator $\mathcal{T}$ is the Dirichlet-to-Neumann operator of the harmonic extension in $B_{\delta}(\mathbf{0})$, that in terms of spherical harmonics is given below.

Assume that we have an expansion of the Dirichlet boundary condition $\left.v\right|_{\partial B_{\delta}(\mathbf{0})}$ as follows

$$
\left.v\right|_{\partial B_{\delta}(\mathbf{0})}(\delta, \theta, \varphi)=\sum_{\ell=0}^{\infty} \sum_{m=-\ell}^{\ell} \alpha_{\ell m} Y_{\ell}^{m}(\theta, \varphi), \quad 0 \leq \theta \leq \pi, 0 \leq \varphi<2 \pi .
$$

Then, we can extend $\left.v\right|_{\partial B_{\delta}(\mathbf{0})}$ harmonically from $\partial B_{\delta}(\mathbf{0})$ to the ball $B_{\delta}(\mathbf{0})$, i.e.,

$$
\left.v\right|_{B_{\delta}(\mathbf{0})}(r, \theta, \varphi)=\sum_{\ell=0}^{\infty} \sum_{m=-\ell}^{\ell} \alpha_{\ell m}\left(\frac{r}{\delta}\right)^{\ell} Y_{\ell}^{m}(\theta, \varphi), \quad 0 \leq r \leq \delta, 0 \leq \theta \leq \pi, 0 \leq \varphi<2 \pi .
$$

Denote by $\mathbf{n}_{\delta}$ the unit normal vector on the sphere $\partial B_{\delta}(\mathbf{0})$ pointing outwards with respect to the ball $B_{\delta}(\mathbf{0})$. As a consequence, we can compute $\partial_{\mathbf{n}_{\delta}} v=\nabla v \cdot \mathbf{n}_{\delta}$ consisting of the normal derivative of $v$ on $\partial B_{\delta}(\mathbf{0})$ :

$$
\mathcal{T} v(\delta, \theta, \varphi):=\partial_{\mathbf{n}_{\delta}} v(\delta, \theta, \varphi)=\sum_{\ell=0}^{\infty} \sum_{m=-\ell}^{\ell} \alpha_{\ell m}\left(\frac{\ell}{\delta}\right) Y_{\ell}^{m}(\theta, \varphi), \quad 0 \leq \theta \leq \pi, 0 \leq \varphi<2 \pi .
$$

It is also easy to see that the bilinear form on the left side of the variational formulation 6.40 is symmetric and coercive due to properties of the Dirichlet-to-Neumann operator $\mathcal{T}$.

\subsubsection{Galerkin discretization}

In order to find basis functions belonging to $H_{0, \delta}^{1}(\mathcal{D})$, we first introduce the radial functions

$$
\varphi_{i}(r)=(1-r) L_{i}^{\prime}\left(\frac{2(r-\delta)}{1-\delta}-1\right)
$$

implying that $\varphi_{i}(1)=0$. Here, $L_{i}$ denotes the Legendre polynomial of $i$-th degree. We then discretize both, the radial part and the spherical part of the unknown $v$, meaning that we represent $v$ by linear combination of the basis functions $\left\{\varphi_{i}(r) Y_{\ell}^{m}(\theta, \varphi)\right\}$ with $1 \leq i \leq N, 0 \leq \ell \leq \ell_{\max }$ and $-\ell \leq m \leq \ell$, where $N$ denotes the maximum degree of Legendre polynomials and $\ell_{\max }$ denotes the maximum degree of spherical harmonics as in Section 6.1. The spanned space of these functions is denoted by $\mathcal{V}_{N, \ell_{\max }}(\mathcal{D})$ which is defined as follows

$$
\mathcal{V}_{N, \ell_{\max }}(\mathcal{D})=\operatorname{span}\left\{\varphi_{i}(r) Y_{\ell}^{m}(\theta, \varphi) \mid 1 \leq i \leq N, 0 \leq \ell \leq \ell_{\max },-\ell \leq m \leq \ell\right\} \subset H_{0, \delta}^{1}(\mathcal{D}) .
$$


Then, we consider a Galerkin discretization of the variational formulation 6.40 that reads: find $\widetilde{v} \in \mathcal{V}_{N, \ell_{\max }}(\mathcal{D})$, such that

$$
\forall \widetilde{w} \in \mathcal{V}_{N, \ell_{\max }}(\mathcal{D}): \quad \int_{\mathcal{D}} \varepsilon_{1} \nabla \widetilde{v} \cdot \nabla \widetilde{w}+\int_{\partial B_{\delta}(\mathbf{0})}(\mathcal{T} \widetilde{v}) \widetilde{w}=\int_{\mathcal{D}} f \widetilde{w} .
$$

Since $\widetilde{v} \in \mathcal{V}_{N, \ell_{\max }}(\mathcal{D})$, we can write $\widetilde{v}$ in the form of

$$
\widetilde{v}(r, \theta, \varphi)=\sum_{i=0}^{N} \sum_{\ell=0}^{\ell_{\max }} \sum_{m=-\ell}^{\ell} v_{i \ell m} \varphi_{i}(r) Y_{\ell}^{m}(\theta, \varphi), \quad \forall \delta \leq r \leq 1,0 \leq \theta \leq \pi, 0 \leq \varphi<2 \pi,
$$

and we consequently have

$$
\left.\mathcal{T} \widetilde{v}\right|_{B_{\delta}(\mathbf{0})}(\delta, \theta, \varphi)=\sum_{i=0}^{N} \sum_{\ell=0}^{\ell_{\max }} \sum_{m=-\ell}^{\ell} v_{i \ell m}\left(\frac{\ell}{\delta}\right) \varphi_{i}(\delta) Y_{\ell}^{m}(\theta, \varphi) .
$$

By substituting 6.45 - 6.46) into 6.44 and taking the test function $\widetilde{w}=\varphi_{i^{\prime}}(r) Y_{\ell^{\prime}}^{m^{\prime}}(\theta, \varphi)$, we then obtain a system of linear equations: $\forall 1 \leq i^{\prime} \leq N, 0 \leq \ell^{\prime} \leq \ell_{\max }, \quad-\ell^{\prime} \leq m^{\prime} \leq \ell^{\prime}$,

$$
\sum_{i=0}^{N} \sum_{\ell=0}^{\ell_{\max }} \sum_{m=-\ell}^{\ell} v_{i \ell m}\left(\int_{\mathcal{D}} \varepsilon_{1} \nabla\left(\varphi_{i} Y_{\ell}^{m}\right) \cdot \nabla\left(\varphi_{i^{\prime}} Y_{\ell^{\prime}}^{m^{\prime}}\right)+\frac{\ell}{\delta} \int_{\partial B_{\delta}(\mathbf{0})} \varphi_{i} Y_{\ell}^{m} \varphi_{i^{\prime}} Y_{\ell^{\prime}}^{m^{\prime}}\right)=\int_{\mathcal{D}} f \varphi_{i^{\prime}} Y_{\ell^{\prime}}^{m^{\prime}} .
$$

In order to write the corresponding system of linear equations, we define an index

$$
k=N\left(\ell^{2}+m+\ell\right)+i \in\left\{1,2, \ldots, N\left(\ell_{\max }+1\right)^{2}\right\}
$$

which corresponds to the triple $(i, \ell, m)$ through a one-to-one mapping between $k$ and $(i, \ell, m)$. Assume that $k$ corresponds to $(i, \ell, m)$ and $k^{\prime}$ corresponds to $\left(i^{\prime}, \ell^{\prime}, m^{\prime}\right)$. Then, we can recast the set of equations (6.47) as a linear system of the form

$$
\mathbf{A} X=F .
$$

Here, $\mathbf{A}$ is a symmetric matrix of dimension $N\left(\ell_{\max }+1\right)^{2} \times N\left(\ell_{\max }+1\right)^{2}$ with elements $(\mathbf{A})_{k k^{\prime}}$, for all $1 \leq k, k^{\prime} \leq N\left(\ell_{\max }+1\right)^{2}$, defined by

$$
(\mathbf{A})_{k^{\prime} k}=\int_{\mathcal{D}} \varepsilon_{1} \nabla\left(\varphi_{i} Y_{\ell}^{m}\right) \cdot \nabla\left(\varphi_{i^{\prime}} Y_{\ell^{\prime}}^{m^{\prime}}\right)+\frac{\ell}{\delta} \int_{\partial B_{\delta}(\mathbf{0})} \varphi_{i} Y_{\ell}^{m} \varphi_{i^{\prime}} Y_{\ell^{\prime}}^{m^{\prime}}
$$

$X$ is the column vector of $N\left(\ell_{\max }+1\right)^{2}$ unknowns $v_{i \ell m}$, i.e.,

$$
(X)_{k}=v_{i \ell m}, \quad \forall 1 \leq k \leq N\left(\ell_{\max }+1\right)^{2}
$$

and $F$ is also a column vector with $N\left(\ell_{\max }+1\right)^{2}$ entities defined by

$$
(F)_{k^{\prime}}=\int_{\mathcal{D}} f \varphi_{i^{\prime}} Y_{\ell^{\prime}}^{m^{\prime}}, \quad \forall 1 \leq k^{\prime} \leq N\left(\ell_{\max }+1\right)^{2} .
$$

In summary, to solve Eq. (6.40), we finally need to solve the linear system (6.48) to obtain all coefficients $v_{i \ell m}$ and then obtain an approximate solution $\widetilde{v}(r, \theta, \varphi) \in \mathcal{V}_{N, \ell_{\max }}(\mathcal{D})$ according to Eq. 6.45). Considering that $v$ is harmonic in $B_{\delta}(\mathbf{0}), \widetilde{v}$ can then be extended harmonically in the ball $\overline{B_{\delta}(\mathbf{0})}$ following 6.42$)$. Therefore, we obtain an approximate solution defined on $B_{1}(\mathbf{0})$ to Eq. 6.39 . 
Remark 6.2. In the global iterative procedure of Section 4.3, we compute the matrix $\mathbf{A}$ of Eq. 6.48 for each subdomain $\Omega_{j}$ a priori, since each $\mathbf{A}$ associated with $\Omega_{j}$ can be reused within the iterative GP-solver for solving Eq. (4.28) and for the outer iterations [1]-[4]. This helps to reduce considerably the cost of the global procedure at the cost of more memory requirements.

\subsubsection{Numerical integration}

In order to implement the method, the integrals in Eq. (6.49) and Eq. (6.51) need to be further computed. We start by observing that the second term in Eq. 6.49 can be simplified

$$
\frac{\ell}{\delta} \int_{\partial B_{\delta}(\mathbf{0})} \varphi_{i} Y_{\ell}^{m} \varphi_{i^{\prime}} Y_{\ell^{\prime}}^{m^{\prime}}=\ell \delta \varphi_{i}(\delta) \varphi_{i^{\prime}}(\delta) \int_{\mathbb{S}^{2}} Y_{\ell}^{m} Y_{\ell^{\prime}}^{m^{\prime}}=\ell \delta \varphi_{i}(\delta) \varphi_{i^{\prime}}(\delta) \delta_{\ell \ell^{\prime}} \delta_{m m^{\prime}}
$$

where $\delta_{\ell \ell^{\prime}}$ and $\delta_{m m^{\prime}}$ are both the Kronecker deltas. As a consequence, the solution matrix $\mathbf{A}$ is symmetric. Next, we present the numerical integration over $\mathcal{D}$ that is used to approximate the integral in the first term in Eq. 6.49) and the integral in Eq. 6.51.

The integral over $\mathcal{D}$ can be divided into two parts: the radial part and spherical part, that is to say, for any given function $h \in L^{1}\left(B_{1}(\mathbf{0})\right)$, we can compute its integral over $\mathcal{D}$ separately as below

$$
\int_{\mathcal{D}} h(\mathbf{x}) \mathrm{d} \mathbf{x}=\int_{\delta}^{1} r^{2} \int_{\mathbb{S}^{2}} h(r, \mathbf{s}) \mathrm{d} \mathbf{s} \mathrm{d} r
$$

where $\mathbf{s} \in \mathbb{S}^{2}$ and $\mathbf{x}=r \mathbf{s}$. To compute the spherical part of this integral, we use again the Lebedev quadrature rule for the unit sphere as in Section 6.1. To integrate the radial part numerically, we use the Legendre-Gauss-Lobatto (LGL) quadrature rule 38 defined by the integration points $x_{m} \in[-1,1]$ and their weights $w_{m}^{\mathrm{lgl}}, 1 \leq m \leq N_{\mathrm{lgl}}$, where $N_{\mathrm{lgl}}$ represents the number of LGL points. Using the change of variable $r=\frac{1-\delta}{2}(x+1)+\delta, x \in[-1,1]$, we approximate the integral by the following quadrature rule

$$
\begin{aligned}
\int_{\mathcal{D}} h(\mathbf{x}) \mathrm{d} \mathbf{x} & =\int_{\delta}^{1} r^{2} \int_{\mathbb{S}^{2}} h(r, \mathbf{s}) \mathrm{d} \mathbf{s} \mathrm{d} r \\
& =\int_{-1}^{1} \frac{1-\delta}{2}\left(\frac{1-\delta}{2}(x+1)+\delta\right)^{2} \int_{\mathbb{S}^{2}} h\left(\frac{1-\delta}{2}(x+1)+\delta, \mathbf{s}\right) \mathrm{d} \mathbf{s} \mathrm{d} x \\
& \approx \frac{1-\delta}{2} \sum_{m=1}^{N_{\mathrm{lgl}}} \sum_{n=1}^{N_{\text {leb }}} w_{m}^{\mathrm{lgl}} w_{n}^{\mathrm{leb}}\left(\frac{1-\delta}{2}\left(x_{m}+1\right)+\delta\right)^{2} h\left(\frac{1-\delta}{2}\left(x_{m}+1\right)+\delta, \mathbf{s}_{n}\right) .
\end{aligned}
$$

The first integral of Eq. 6.49 and the integral of Eq. 6.51 can then be numerically computed using this quadrature. See Figure 7 for a schematic diagram of the Lebedev points and the LGL points.

\section{Numerical results}

In this section, we present some numerical results of the proposed method for solving the PDEs 4.17 - 4.18). Before investigating a realistic solute molecule, we will start by testing the GP-solver for the unit sphere. We then consider small molecules and compute the electrostatic contribution to 

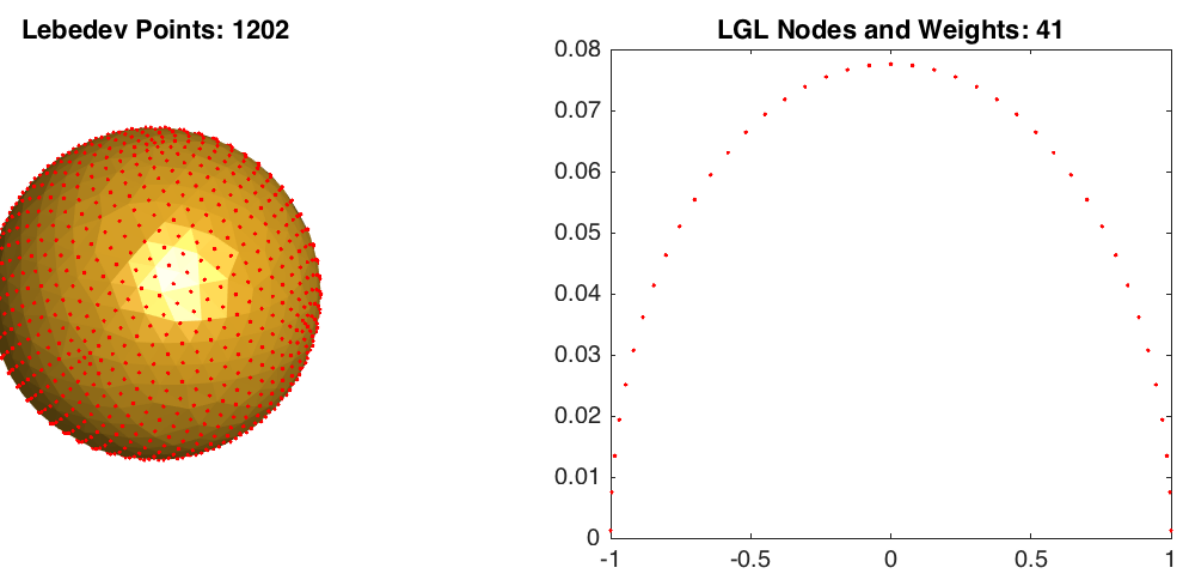

Figure 7: Schematic diagram of the Lebedev points (left) and the LGL points as well as their weights (right).

the solvation energy to study its dependency with respect to numerous parameters. In the following tests, we simply choose a polynomial function to be $\xi$ in Eq. (3.7) as follows

$$
\xi(t)=1+\left(\varepsilon_{\mathrm{s}}-1\right) t^{3}(10+3 t(-5+2 t)), \quad 0 \leq t \leq 1,
$$

which satisfies the previously-mentioned conditions (3.8) at $t=0$ and $t=1$. This function does not come from experimental fitting nor have a physical meaning, but taken for the numerical tests of the proposed method only.

\subsection{GP-solver test}

In Section 6.2, we present the GP-solver in the unit ball, which will be called repeatedly in the domain decomposition scheme. In this part, we first test the GP-solver for solving Eq. 6.37) to have a first look at the solver itself. For the sake of simplicity, we assume here that the dielectric permittivity function $\varepsilon$ only depends on the radial variable $r$, i.e., the dielectric permittivity is symmetric. Let $r_{1}=0.4, r_{\mathrm{p}}=0.3, r_{0}=0.3$ and define the dielectric permittivity function $\varepsilon(r)$ by

$$
\varepsilon(r)= \begin{cases}1 & \text { if } 0 \leq r \leq r_{1}, \\ \xi\left(\frac{r-r_{1}}{r_{\mathrm{p}}+r_{0}}\right) & \text { if } r_{1} \leq r \leq 1, \\ \varepsilon_{\mathrm{s}} & \text { if } r \geq 1,\end{cases}
$$

where $\varepsilon_{\mathrm{s}}=10$. Assuming that the sphere carries a point charge 1 at its center, i.e., $q_{1}=1$, we take $\Phi=\frac{1}{r}, f_{1}=-\frac{\varepsilon^{\prime}(r)}{r^{2}}$ and $\phi_{1}=-1$ in Eq. 6.37). Since both the dielectric permittivity function and the solution only depend on the radial variable, the discretization of the spherical part can be neglected, i.e., $\ell_{\max }=0$. To have a good approximation in the radial direction, we set a high maximum degree of Legendre polynomials $N=20$ and a large number of LGL points $N_{\text {lgl }}=200$. By running the GP-solver, we then obtain Figure 8 illustrating the dielectric permittivity $\varepsilon(r)$ and the numerical solution $u_{1}(r)$ to Eq. 6.37). It is observed that $u_{1}(r)$ is constant in $\left[0, r_{1}\right]$ because of the harmonicity. 

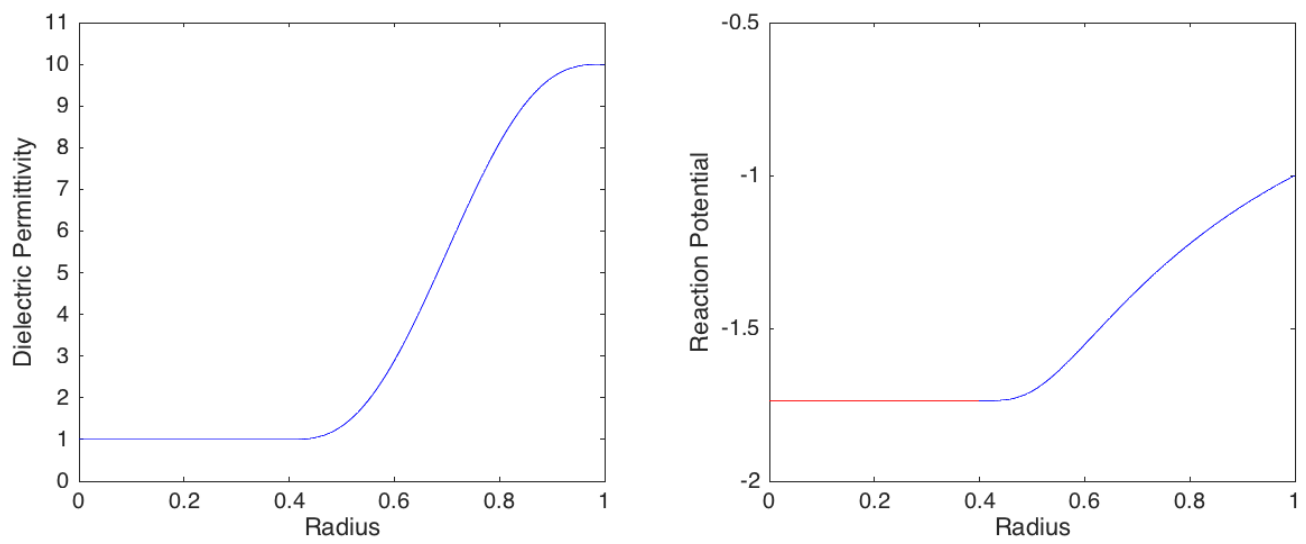

Figure 8: The left figure illustrates the dielectric permittivity function $\varepsilon(r)$; the right plots the numerical solution to Eq. 6.37). Only the blue part is subject to the computational domain, the red part of the curve depicts the harmonic extension.

\subsection{Convergence of the global strategy}

With the domain decomposition method using the GP-solver for solving Eq. 4.28) and the Laplace solver for solving (4.29), we can then solve numerically Eqs 4.26 -4.27) following the iterative procedure presented in Section 4.3 .

The Schwarz domain decomposition method (for solving the PDE (4.28) and (4.29) is wellstudied and its convergence can be guaranteed [46]. To study the convergence of the proposed method with respect to the number of outer iterations, we first take the example of formaldehyde. The following discretization parameters are used: the maximum degree of spherical harmonics $\ell_{\max }=11$, the number of Lebedev points $N_{\text {leb }}=1202$, the maximum degree of Legendre polynomials $N=15$, the number of LGL nodes $N_{\mathrm{lgl}}=50$. These parameters are given based on a further study in Section 7.6. Furthermore, we take the solvent permittivity $\varepsilon_{\mathrm{s}}=78.4$ (water, at room temperature $25^{\circ} \mathrm{C}$ ), the solvent probe radius $r_{\mathrm{p}}=1.5 \AA$ and $r_{0}=1 \AA$.

We use the convention that in the numerical tests, the stopping criterion Tol is set to $10^{-7}$ by default. In this article, the unit of energy is set to $\mathrm{e}^{2} / \AA=332.06364 \mathrm{kcal} / \mathrm{mol}$ (see [25, Section 1.4] for this equality), where e denotes the elementary charge.

In addition, we use another convention that at each outer iteration of the global strategy, the number of inner iterations for solving the GP-equation (4.28) is fixed to be 8 . This number is determined empirically and it allows us to obtain an accurate enough numerical solution to Eq. 4.28 at each outer iteration.

The error of the electrostatic contribution is computed as follows

$$
\operatorname{Error}\left(N_{\mathrm{it}}\right)=\left|E_{N_{\mathrm{it}}}^{\mathrm{s}}-E_{\infty}^{\mathrm{s}}\right|
$$

where $E_{N_{\mathrm{it}}}^{\mathrm{s}}$ is the electrostatic contribution computed at the $N_{\mathrm{it}}$-th outer iteration in Section 4.3 and the "exact" electrostatic contribution $E_{\infty}^{\mathrm{s}}$ is obtained after 15 outer iterations (this number is large enough) with the same discretization parameters as mentioned above. In Figure 9 , which illustrates the numerical electrostatic contribution to the solvation energy of formaldehyde with respect to the 

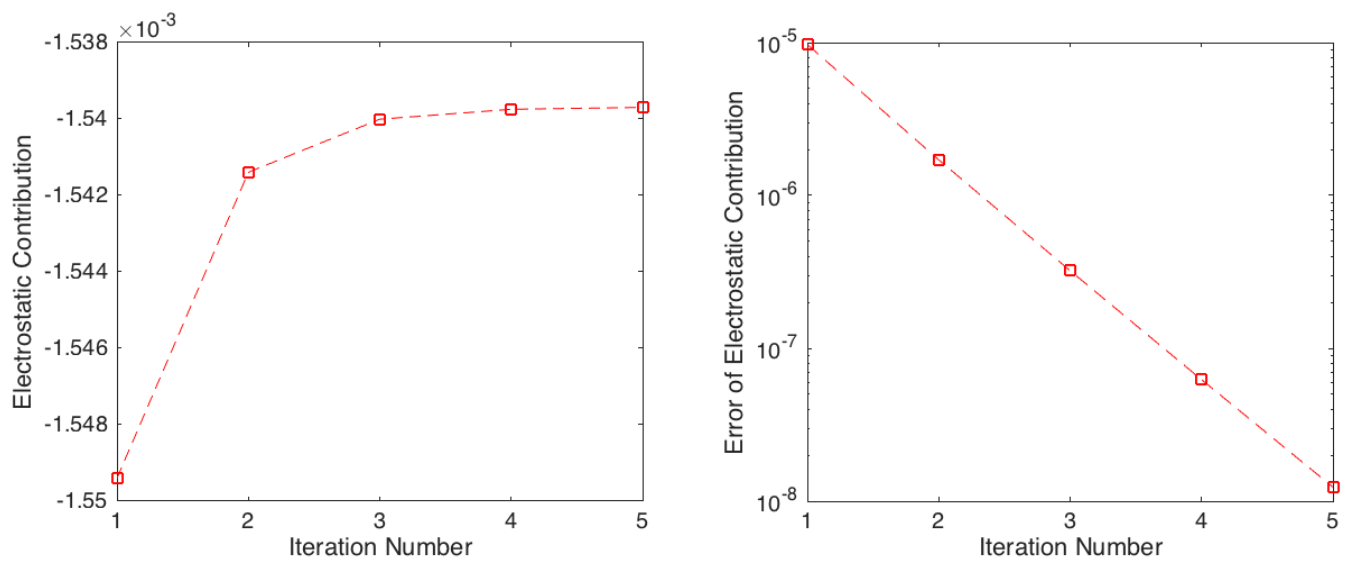

Figure 9: On the left, the curve illustrates the computed electrostatic contribution to the solvation energy of formaldehyde with respect to $N_{\mathrm{it}}$; on the right, the curve illustrates the error of the electrostatic contribution with respect to $N_{\text {it }}$. The following parameters are used: $\ell_{\max }=11, N_{\text {leb }}=$ $1202, N=15, N_{\mathrm{lgl}}=50, \varepsilon_{\mathrm{s}}=78.4, r_{\mathrm{p}}=1.5 \AA, r_{0}=1 \AA$.

number of outer iterations $N_{\mathrm{it}}$, it is observed that the error of electrostatic contribution converges geometrically with respect to $N_{\text {it }}$ and the procedure stops at $N_{\text {it }}=5$ when the stopping criterion is reached, i.e., $\left|E_{N_{\mathrm{it}}}^{\mathrm{s}}-E_{N_{\mathrm{it}}-1}^{\mathrm{s}}\right|<\mathrm{Tol}$.

Then, we present the example of a larger molecule, the caffeine consisting of 24 atoms, with the parameters $\ell_{\max }=9, N_{\text {leb }}=350, N=15, N_{\mathrm{lgl}}=30, \varepsilon_{\mathrm{s}}=78.4, r_{\mathrm{p}}=1.5 \AA, r_{0}=1 \AA$. The discretization parameters are taken smaller than those for formaldehyde because of the running time. This does not matter since we only want to study the convergence of the method with respect to the number of outer iterations. As above, the "exact" electrostatic contribution $E_{\infty}^{\mathrm{s}}$ is obtained after 15 outer iterations and the error is computed from (7.57). As can be seen in Figure 10, the error of electrostatic contribution converges also geometrically with respect to $N_{\text {it }}$.

\subsection{Graphical illustration of the reaction potential}

We give here some graphical illustration of the reaction potential in the enlarged cavity $\Omega_{0}$ and on the SES. In Figure 11, we illustrate the reaction potential $W$ of hydrogen-fluoride and the magnitude of the corresponding reaction field (i.e., $\nabla W$ ). In Figure 12 and 13 , we illustrate the reaction potential of hydrogen-fluoride, formaldehyde and caffeine in water. The rotational symmetry of hydrogen-fluoride and the mirror symmetry of formaldehyde are observed as expected.

\subsection{Thickness of the intermediate layer}

We then draw the attention to the influence of the thickness of the intermediate layer, which is given by $r_{\mathrm{p}}+r_{0}$. For the sake of simplicity, we fix the parameter $r_{0}=0 \AA$, implying that $\Gamma_{0}$ and $\Gamma_{\text {sas }}$ coincide. In the ddPCM algorithm presented in [47, the dielectric permittivity is discontinuous across the solute-solvent boundary and jumps from 1 to $\varepsilon_{\mathrm{s}}$. In the following test, we take $\Gamma_{0}$ as the solute-solvent boundary in the ddPCM algorithm and expect that the numerical electrostatic 

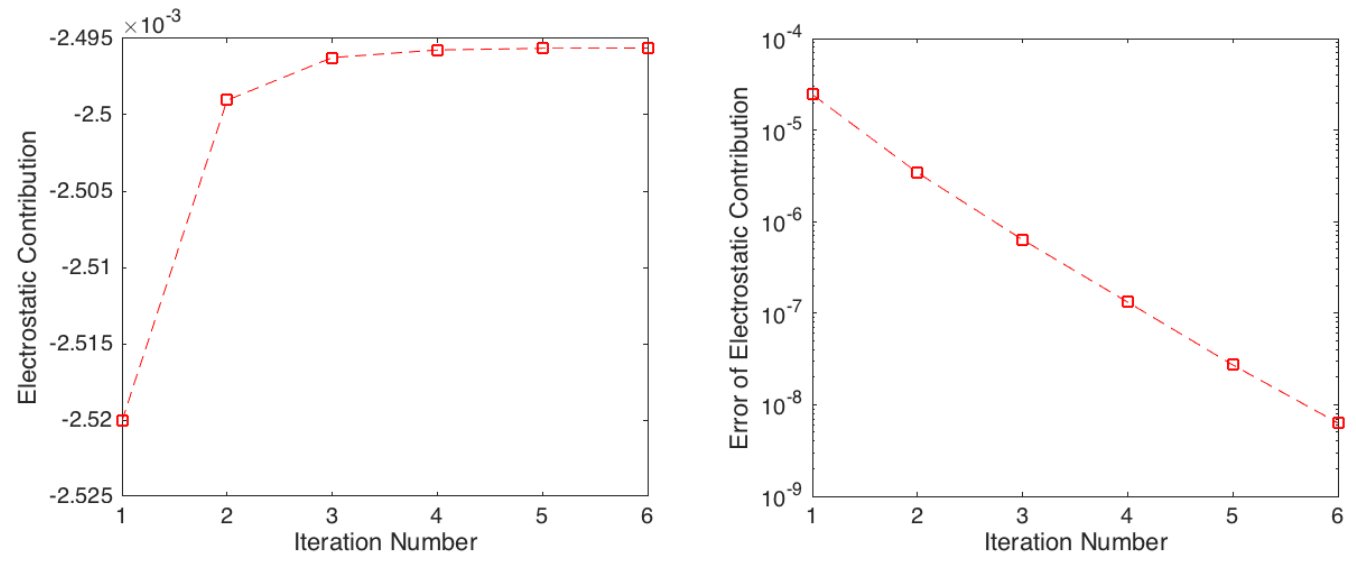

Figure 10: On the left, the curve illustrates the numerical electrostatic contribution to the solvation energy of caffeine with respect to $N_{\text {it }}$; on the right, the curve illustrates the error of the electrostatic contribution with respect to $N_{\text {it }}$. The following parameters are used: $\ell_{\max }=9, N_{\text {leb }}=350, N=$ $15, N_{\mathrm{lgl}}=30, \varepsilon_{\mathrm{s}}=78.4, r_{\mathrm{p}}=1.5 \AA, r_{0}=1 \AA$.
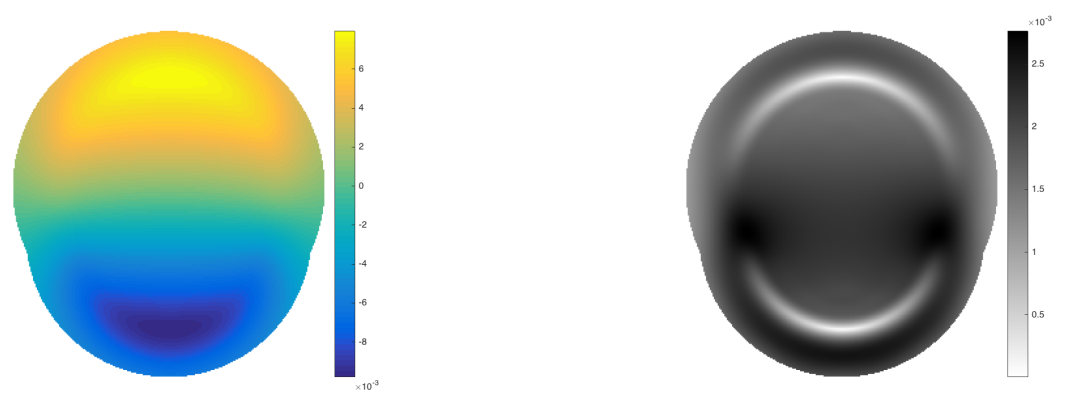

Figure 11: The reaction potential (left) and the magnitude of the corresponding reaction field (right) in the YZ-plane for the hydrogen-fluoride molecule with two atoms, with the parameters $\ell_{\max }=11, N_{\text {leb }}=1202, N=15, N_{\text {lgl }}=50, \varepsilon_{\mathrm{s}}=2, r_{\mathrm{p}}=1.5 \AA, r_{0}=1 \AA$. The colorbars represent respectively the reaction potential value and the magnitude of the reaction field. 

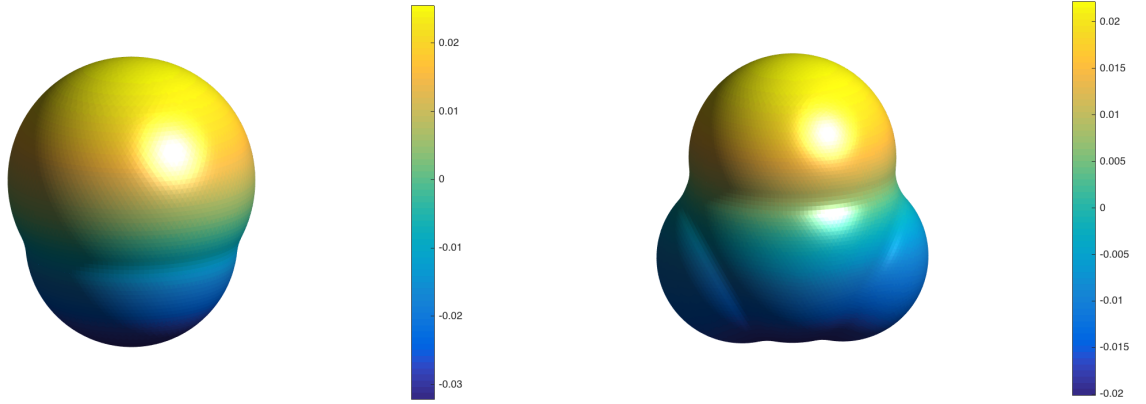

Figure 12: Reaction potential of hydrogen-fluoride and formaldehyde on the SES, both computed with the parameters $\ell_{\max }=11, N_{\text {leb }}=1202, N=15, N_{\text {lgl }}=50, \varepsilon_{\mathrm{s}}=78.4, r_{\mathrm{p}}=1.5 \AA, r_{0}=1 \AA$.

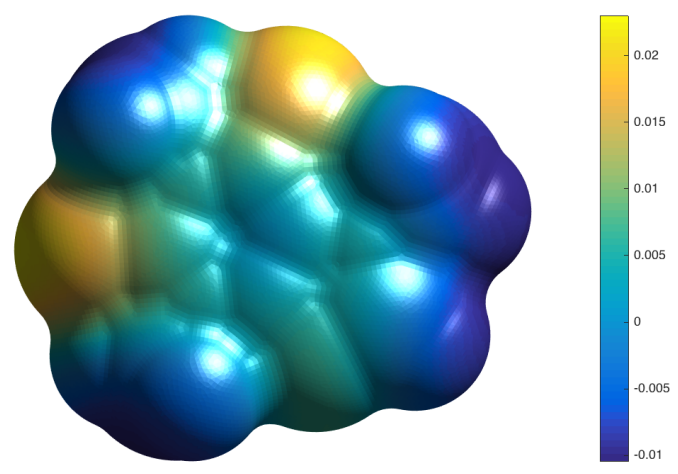

Figure 13: Reaction potential of caffeine on the SES, computed with the parameters $\ell_{\max }=$ $9, N_{\mathrm{leb}}=350, N=15, N_{\mathrm{lgl}}=30, \varepsilon_{\mathrm{s}}=78.4, r_{\mathrm{p}}=1.5 \AA, r_{0}=1 \AA$. 


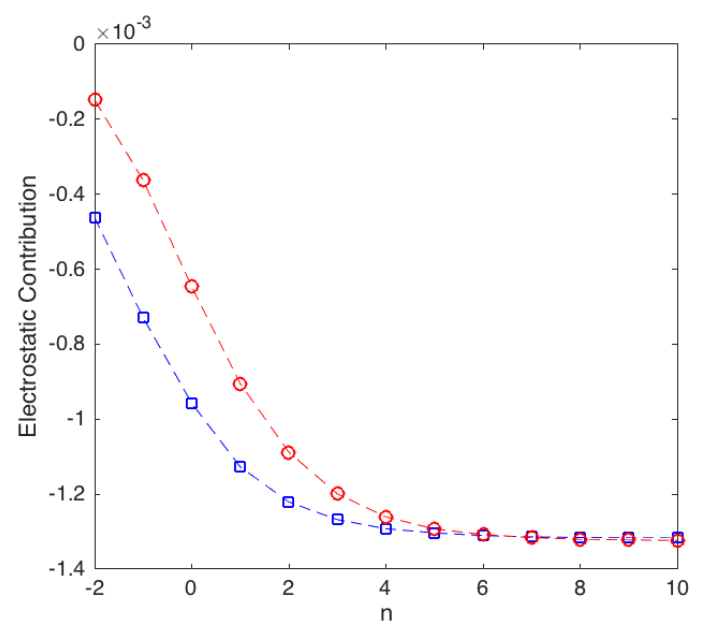

Figure 14: The blue curve plots the electrostatic contribution of hydrogen-fluoride with respect to $n$ (the layer thickness $2^{-n} \AA$ ), computed from the proposed algorithm with the parameters $\ell_{\max }=$ $11, N_{\text {leb }}=1202, N=15, N_{\mathrm{lgl}}=50, \varepsilon_{\mathrm{s}}=2$; the red curve plots the results computed from the ddPCM algorithm with the same parameters of spherical harmonics $\ell_{\max }=11, N_{\text {leb }}=1202$ and the same solvent permittivity $\varepsilon_{\mathrm{s}}=2$.

contribution to the solvation energy computed from our method tends to the one computed from the ddPCM algorithm as the thickness of the intermediate layer $\mathcal{L}$ tends to zero, i.e., $r_{\mathrm{p}} \rightarrow 0$. This implies that both, $\Gamma_{\text {ses }}$ and $\Gamma_{0}$, tend to the VdW-surface.

To verify this, we take again the example of hydrogen-fluoride. Figure 14 illustrates the numerical electrostatic contribution to the solvation energy with respect to $n$ where the thickness of the intermediate layer is parametrized by $r_{\mathrm{p}}=2^{-n} \AA$. We observe that the electrostatic contributions from both algorithms almost tend to coincide when the layer vanishes. This means that the proposed method is consistent with the ddPCM in this case.

\subsection{Solvent dielectric constant}

We illustrate in Figure 15 how the electrostatic contribution to the solvation energy and the total number of outer iterations varies with respect to different solvent permittivities $\varepsilon_{\mathrm{s}}=2^{k}, k=$ $1,2, \ldots, 15$. We observe that the numerical electrostatic contribution to the solvation energy varies smoothly and converges to some quantity for increasing solvent permittivities $\varepsilon_{\mathrm{s}}$. Another interesting observation is that when $\varepsilon_{\mathrm{s}}$ becomes large, the total number of outer iterations tends to decrease. This can be explained by the fact that the solvent is conductor-like and consequently, the initial guess of potential $g^{0}$ is very accurate for high permittivities $\varepsilon_{\mathrm{s}}$.

\subsection{Discretization parameters}

We consider the dichloromethane solvent $\left(\varepsilon_{\mathrm{s}}=8.93\right)$ at room temperature $25^{\circ} \mathrm{C}$. First of all, we compute numerically the "exact" electrostatic contribution to the solvation energy of hydrogenfluoride, denoted by $E_{\text {exact }}^{\mathrm{s}}$, with large discretization parameters $\ell_{\max }=20, N_{\text {leb }}=4334, N=$ 

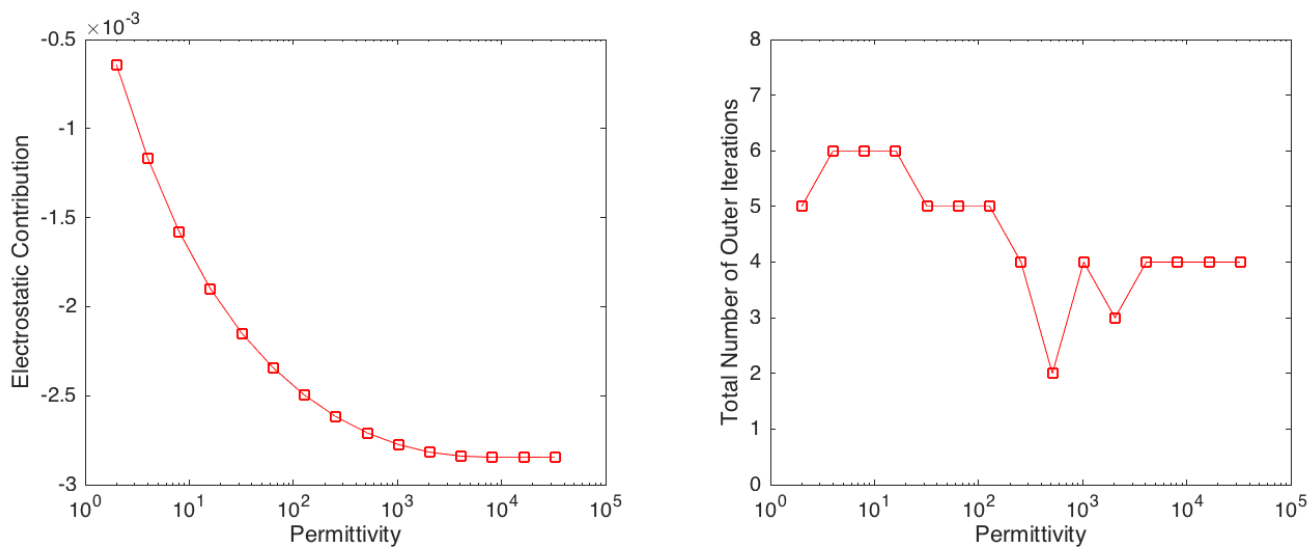

Figure 15: The left figure shows the electrostatic contribution to the solvation energy of hydrogenfluoride when the solvent dielectric constant $\varepsilon_{\mathrm{s}}$ increases; the right figure plots the total number of outer iterations to reach the stopping criterion Tol with respect to $\varepsilon_{\mathrm{s}}$. The following parameters are used: $\ell_{\max }=11, N_{\text {leb }}=1202, N=20, N_{\text {lgl }}=50, r_{\mathrm{p}}=1.5 \AA, r_{0}=1 \AA$.

$25, N_{\mathrm{lgl}}=50$ and the other parameters $r_{\mathrm{p}}=1.5 \AA, r_{0}=1 \AA$. We treat $E_{\text {exact }}^{\mathrm{s}}$ as the benchmark of the electrostatic contribution to the solvation energy.

We then illustrate how the electrostatic contribution to the solvation energy of hydrogen-fluoride varies respectively with respect to the maximum degree $\ell_{\max }$ of spherical harmonics and to the number $N_{\text {leb }}$ of Lebedev points in the dichloromethane solvent. On the left of Figure 16, we report the electrostatic contribution with respect to $\ell_{\max }$ which varies from 3 to 15 . Further, on the right of Figure 16, we report the electrostatic contribution with respect to $N_{\text {leb }}$ which varies from 350 to 4334 . From the left figure, we observe that the proposed algorithm provides systematically improvable approximations when the parameters of spherical harmonics increases.

Similarly as above, we now illustrate how the electrostatic contribution to the solvation energy of hydrogen-fluoride varies with respect to the maximum degree $N$ of Legendre polynomials and to the number $N_{\mathrm{lgl}}$ of LGL points in the dichloromethane solvent. On the left of Figure 17, we report the electrostatic contribution with respect to $N$ varying from 6 to 20 . Further, on the right of Figure 17, we report the electrostatic contribution with respect to $N_{\operatorname{lgl}}$ varying from 20 to 80 . Again, it is observed that the proposed algorithm provides asymptotically systematically improvable approximations when the discretization of Legendre polynomials increases. These results help us to get a know-how in order to select, for a given molecule, the proper choice of discretization parameters $\ell_{\max }, N_{\text {leb }}, N, N_{\text {lgl }}$ for an acceptable accuracy.

\subsection{Robustness with respect to geometrical parameters}

We now study in Figure 18 the variation of the electrostatic contribution to the solvation energy of hydrogen-fluoride while rotating the fluoride atom around the hydrogen atom. We observe that the variation in the electrostatic contribution, which should be invariant with respect to the rotation angle, is systematically controlled. For the two sets of parameters, see the caption of Figure 18, the variation is around $1.5 \%$ and $0.25 \%$ respectively. 

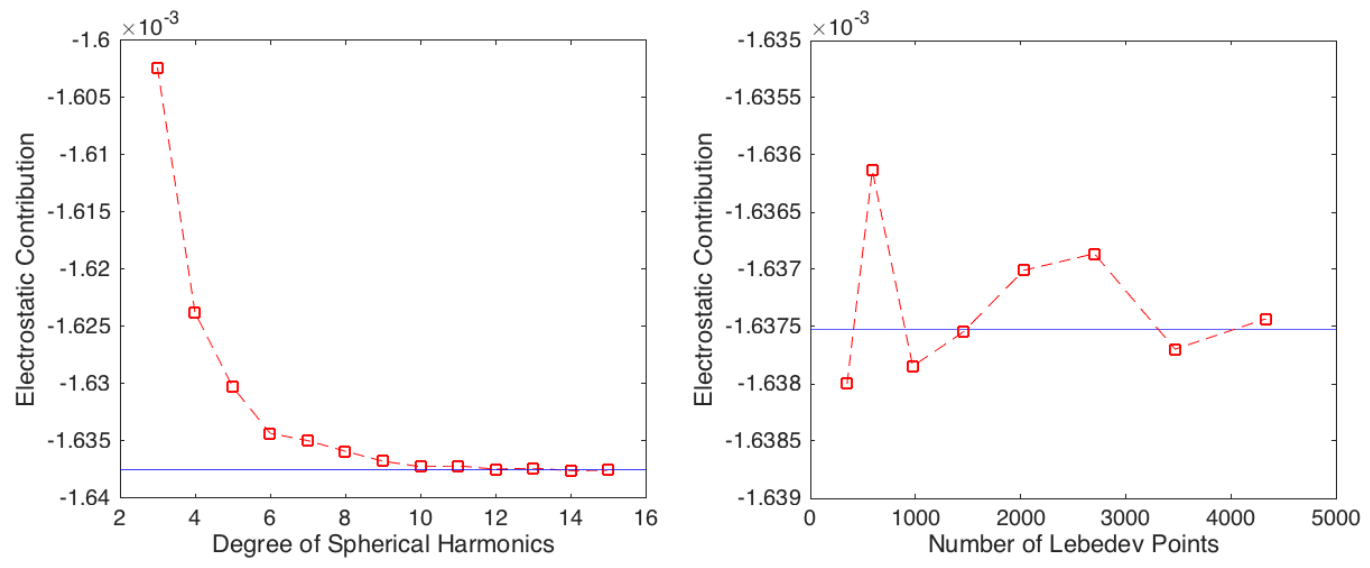

Figure 16: The left figure plots the electrostatic contribution to the solvation energy of hydrogenfluoride with respect to $\ell_{\max }$ when $N_{\text {leb }}$ is set to 1454. The right figure plots the electrostatic contribution with respect to $N_{\text {leb }}$ when $\ell_{\max }$ is set to 15 . In both figures, the blue line represents the "exact" electrostatic contribution $E_{\text {exact }}^{\mathrm{s}}$. In addition, the following parameters are used: $N=$ $20, N_{\mathrm{lgl}}=50, \varepsilon_{\mathrm{s}}=8.93, r_{\mathrm{p}}=1.5 \AA, r_{0}=1 \AA$.
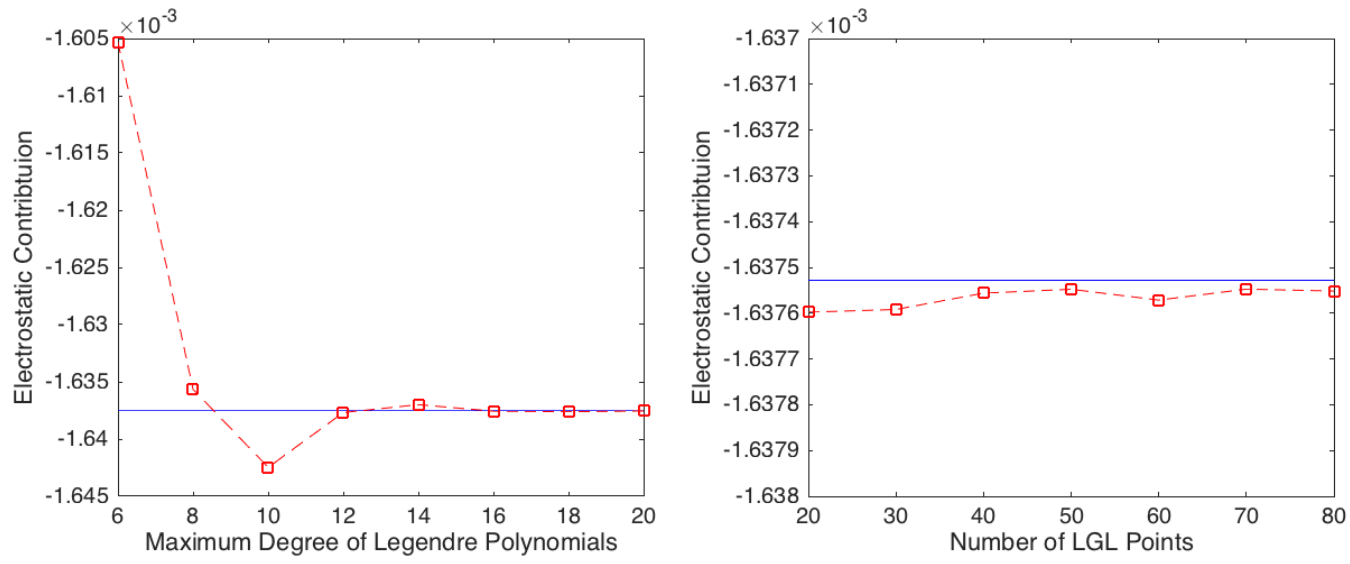

Figure 17: The left figure plots the electrostatic contribution to the solvation energy of hydrogenfluoride with respect to $N$ when $N_{\mathrm{lgl}}$ is set to 50. The right figure plots the electrostatic contribution with respect to $N_{\operatorname{lgl}}$ when $N$ is set to 20 . In both figures, the blue line represents the "exact" electrostatic contribution $E_{\text {exact }}^{\mathrm{s}}$. In addition, the following parameters are used: $\ell_{\max }=15, N_{\text {leb }}=$ $1454, \varepsilon_{\mathrm{s}}=8.93, r_{\mathrm{p}}=1.5 \AA, r_{0}=1 \AA$. 


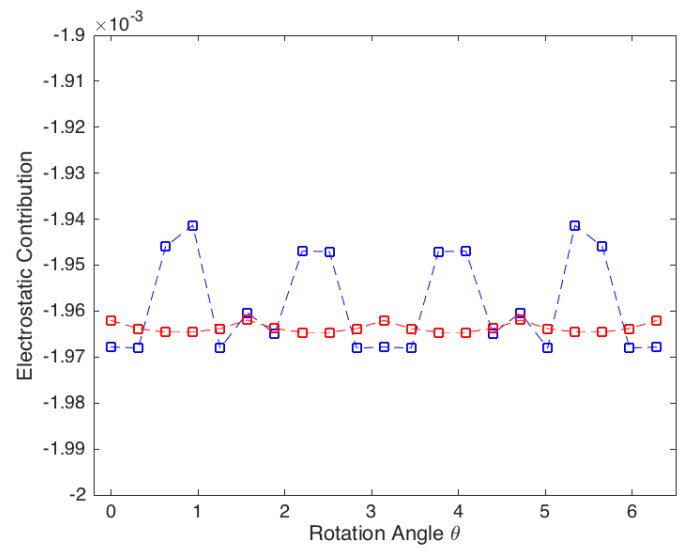

Figure 18: The variation of the electrostatic contribution to the solvation energy of hydrogenfluoride with respect to the angle of rotating the flouride atom around the hydrogen atom. The blue curve corresponds to the parameters $\ell_{\max }=7, N_{\text {leb }}=86, N=10, N_{\text {lgl }}=20$, the red curve corresponds to the parameters $\ell_{\max }=11, N_{\text {leb }}=1202, N=20, N_{\text {lgl }}=40$. Furthermore, the following parameters are used: $\varepsilon_{\mathrm{s}}=8.93, r_{\mathrm{p}}=1.5 \AA, r_{0}=1 \AA$.

Further, we study the electrostatic contribution to the solvation energy under a dissociation of hydrogen-fluoride. We vary the separation distance between the hydrogen atom and the fluoride atom from $8.96 \AA$ to $9.36 \AA$ where the topology of the SES changes in the sense that the SES becomes two disconnected subsurfaces, see Figure 19 for a geometrical illustration. The left plot of Figure 20 illustrates that the energy profile is completely smooth when the topology of the SES changes. The right figure of Figure 20 provides the electrostatic contribution to the solvation energy when the separation distance varies in a wider range from $1.77 \AA$ to $9.37 \AA$.

We next study the smoothness of the numerical electrostatic contribution to the solvation energy of formaldehyde with respect to a topological change in the sense that the nature of the patches of the SES changes. We choose the coordinate system so that all nuclei lie in the $y z$-plane and that carbon and oxygen atoms have $y=0$ coordinate. We then move the $z$-coordinate of the two hydrogen atoms further away from the carbon and oxygen atoms, see Figure 21 for an illustration. Figure 22 plots the numerical electrostatic contribution to the solvation energy with respect to the downwards displacement of the two hydrogen atoms. On the left, we plot the numerical electrostatic contribution with respect to displacement of the two hydrogen atoms in a large range from $0 \AA$ to $1 \AA$. On the right, we plot the numerical electrostatic contribution in the neighborhood of the threshold when the concave SES patches, marked in blue in Figure 21, first appear and we observe that the energy-profile is approximately smooth.

\section{Conclusion}

We have proposed a construction to deal with a continuous permittivity function $\varepsilon(\mathbf{x})$ for the SESbased PCM, in which a switching region on the solute-solvent boundary is introduced, using the signed distance function to the SAS. The switching region potentially also allows one to describe local effects close to the solute-solvent interface. Further, to solve this SES-based PCM, we lay out 

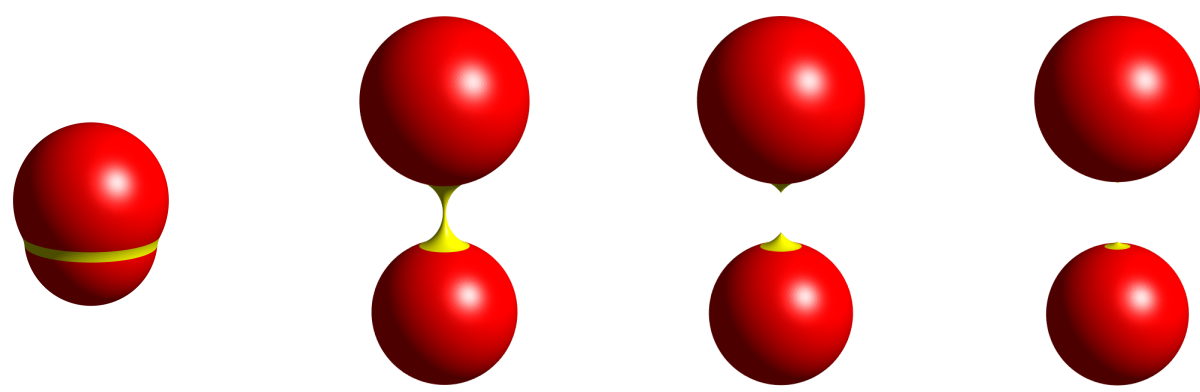

Figure 19: Topological change of the SES of hydrogen-fluoride when the distance between the two atomic centers increases. The most left figure illustrates the SES of hydrogen-fluoride in equilibrium and the three figures from the left to right corresponds to the distances between the centers increased respectively to $8.96 \AA, 9.16 \AA$ and $9.36 \AA$.
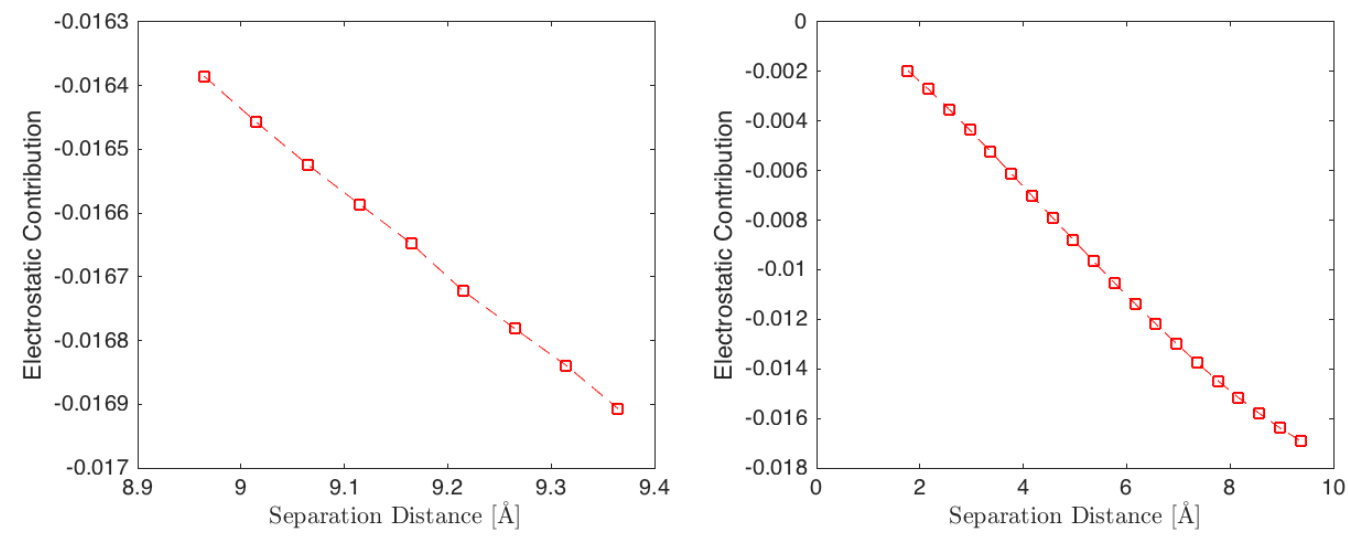

Figure 20: Electrostatic contribution of hydrogen-fluoride with respect to the separation distance between the atomic centers with parameters $\ell_{\max }=11, N_{\mathrm{leb}}=1202, N=20, N_{\mathrm{lgl}}=40, \varepsilon_{\mathrm{s}}=$ $8.93, r_{\mathrm{p}}=1.5 \AA, r_{0}=0 \AA$. On the left, the separation distance varies between $8.96 \AA$ and $9.36 \AA$ where the topology of the SES changes as showed in Figure 19 . On the right, the separation distance varies in a larger range. 

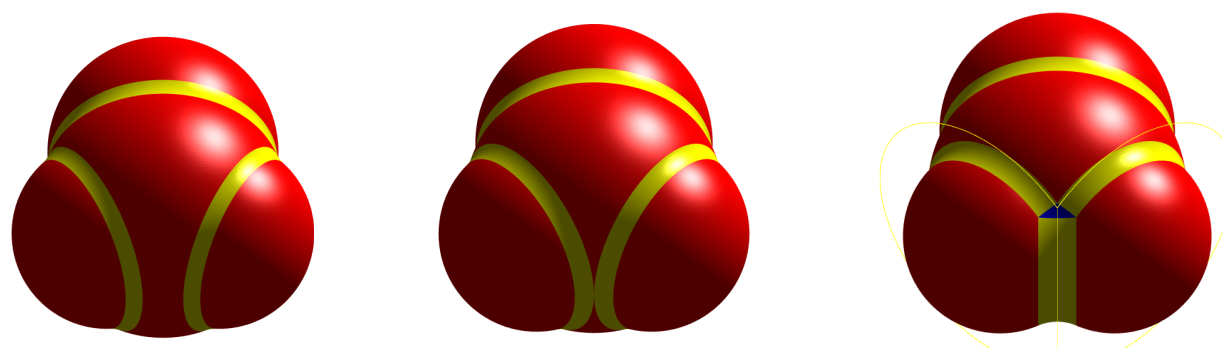

Figure 21: Different SESs of formaldehyde when the two hydrogen atoms displace downwards. The left figure illustrates the SES of formaldehyde in equilibrium; the middle figure illustrates the SES when concave patches are about to appear; the right figure illustrates the SES when the concave patches (in blue) have appeared.
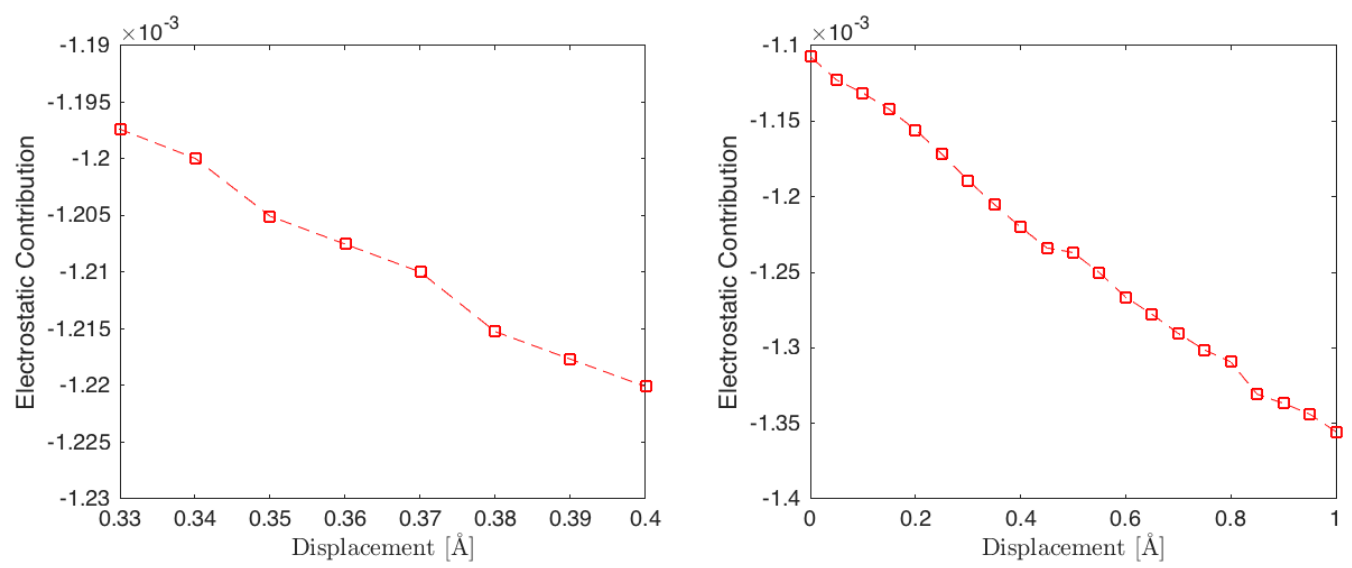

Figure 22: Electrostatic contribution to the solvation energy of formaldehyde with respect to the displacement of the two hydrogen atoms with parameters $\ell_{\max }=11, N_{\text {leb }}=1454, N=15, N_{\text {lgl }}=$ $50, \varepsilon_{\mathrm{s}}=8.93, r_{\mathrm{p}}=1.5 \AA, r_{0}=1 \AA$. On the left, the displacement varies near the threshold when the concave SES patches first appear as showed in the middle of Figure 21. On the right, the displacement varies from $0 \AA$ to $1 \AA$. 
a mathematical framework to compute the solution on an unbounded domain that only involves computing problems in a bounded domain (the enlarged cavity $\Omega_{0}$ ). For each problem in the enlarged cavity, we propose to use the Schwarz domain decomposition method where the global problem is divided into simple sub-problems each defined in a ball. We provided numerical tests to illustrate the resulting energy profile is smooth with respect to geometrical parameters and that the energy is systematically improvable.

We focused here on the modeling part and the resulting discretization method. This work is meant to study the feasibility to solve a SES-based solvation model using a domain decomposition method and analyzing its properties. An upcoming paper will focus on an efficient implementation in Fortran which is a work in progress.

\section{Acknowledgement}

We would like to thank Pacal Frey, Eric Cancès, Filippo Lipparini and Benedetta Mennucci for the fruitful discussion about meshing molecular surfaces and constructing the polarizable continuum model.

Chaoyu Quan would like to acknowledge the support from the Fondation Sciences Mathématiques de Paris.

Benjamin Stamm acknowledges the funding from the German Academic Exchange Service (DAAD) from funds of the "Bundesministeriums für Bildung und Forschung" (BMBF) for the project Aa-Par-T (Project-ID 57317909).

Yvon Maday and Chaoyu Quan acknowledge the funding from the PICS-CNRS and the PHC PROCOPE 2017 (PROJET N 37855ZK).

\section{References}

[1] Adams, R. A. and Fournier, J. J. (2003). Sobolev spaces, volume 140. Academic Press.

[2] Aksoylu, B., Bond, S. D., Cyr, E. C., and Holst, M. (2012). Goal-oriented adaptivity and multilevel preconditioning for the Poisson-Boltzmann equation. Journal of Scientific Computing, $52(1): 202-225$.

[3] Baker, N. A., Sept, D., Joseph, S., Holst, M. J., and McCammon, J. A. (2001). Electrostatics of nanosystems: application to microtubules and the ribosome. Proceedings of the National Academy of Sciences, 98(18):10037-10041.

[4] Bardhan, J. P. (2009). Numerical solution of boundary-integral equations for molecular electrostatics. Journal of Chemical Physics, 130(9):094102.

[5] Basilevsky, M. V., Grigoriev, F. V., Nikitina, E. A., and Leszczynski, J. (2010). Implicit electrostatic solvent model with continuous dielectric permittivity function. Journal of Physical Chemistry B, 114(7):2457-2466.

[6] Beentjes, C. H. (2015). Quadrature on a spherical surface. Working note available on the website http://people.maths.ox.ac.uk/beentjes/Essays.

[7] Boschitsch, A. H., Fenley, M. O., and Zhou, H.-X. (2002). Fast boundary element method for the linear Poisson-Boltzmann equation. Journal of Physical Chemistry B, 106(10):2741-2754. 
[8] Bugeanu, M., Di Remigio, R., Mozgawa, K., Reine, S. S., Harbrecht, H., and Frediani, L. (2015). Wavelet formulation of the polarizable continuum model. II. Use of piecewise bilinear boundary elements. Physical Chemistry Chemical Physics, 17:31566-31581.

[9] Cammi, R. and Mennucci, B. (2007). Continuum Solvation Models in Chemical Physics: From Theory to Applications. John Wiley.

[10] Cancès, E., Maday, Y., and Stamm, B. (2013). Domain decomposition for implicit solvation models. Journal of Chemical Physics, 139(5):054111.

[11] Cancès, E. and Mennucci, B. (1998). New applications of integral equations methods for solvation continuum models: ionic solutions and liquid crystals. Journal of Mathematical Chemistry, $23(3-4): 309-326$.

[12] Cancès, E., Mennucci, B., and Tomasi, J. (1997). A new integral equation formalism for the polarizable continuum model: Theoretical background and applications to isotropic and anisotropic dielectrics. Journal of Chemical Physics, 107(8):3032-3041.

[13] Ciaramella, G. and Gander, M. J. (2016). Analysis of the parallel Schwarz method for growing chains of fixed-size subdomains: part I. Preprint. To appear in SIAM Journal on Numerical Analysis.

[14] Connolly, M. L. (1983). Analytical molecular surface calculation. Journal of Applied Crystallography, 16(5):548-558.

[15] Davis, M. E., Madura, J. D., Luty, B. A., and McCammon, J. A. (1991). Electrostatics and diffusion of molecules in solution: simulations with the University of Houston Brownian dynamics program. Computer Physics Communications, 62(2-3):187-197.

[16] Davis, M. E. and McCammon, J. A. (1990). Electrostatics in biomolecular structure and dynamics. Chemical Reviews, 90(3):509-521.

[17] Dolinsky, T. J., Czodrowski, P., Li, H., Nielsen, J. E., Jensen, J. H., Klebe, G., and Baker, N. A. (2007). PDB2PQR: expanding and upgrading automated preparation of biomolecular structures for molecular simulations. Nucleic Acids Research, 35(Web Server issue):W522-W525.

[18] Fisicaro, G., Genovese, L., Andreussi, O., Marzari, N., and Goedecker, S. (2016). A generalized Poisson and Poisson-Boltzmann solver for electrostatic environments. Journal of Chemical Physics, 144(1):014103.

[19] Grant, J. A., Pickup, B. T., and Nicholls, A. (2001). A smooth permittivity function for Poisson-Boltzmann solvation methods. Journal of Computational Chemistry, 22(6):608-640.

[20] Griffiths, D. (2008). Introduction to elementary particles. John Wiley \& Sons.

[21] Hackbusch, W. (2012). Integral equations: theory and numerical treatment, volume 120. Birkhäuser.

[22] Harbrecht, H. and Randrianarivony, M. (2011). Wavelet BEM on molecular surfaces: solvent excluded surfaces. Computing, 92(4):335-364. 
[23] Haxton, D. J. (2007). Lebedev discrete variable representation. Journal of Physics B: Atomic, Molecular and Optical Physics, 40(23):4443.

[24] Holst, M., Mccammon, J. A., Yu, Z., Zhou, Y., and Zhu, Y. (2012). Adaptive finite element modeling techniques for the Poisson-Boltzmann equation. Communications in Computational Physics, 11(1):179-214.

[25] Holst, M. J. et al. (1994). The Poisson-Boltzmann equation: Analysis and multilevel numerical solution.

[26] Im, W., Beglov, D., and Roux, B. (1998). Continuum solvation model: computation of electrostatic forces from numerical solutions to the Poisson-Boltzmann equation. Computer Physics Communications, 111(1):59-75.

[27] Jurrus, E., Engel, D., Star, K., Monson, K., Brandi, J., Felberg, L. E., Brookes, D. H., Wilson, L., Chen, J., Liles, K., et al. (2017). Improvements to the APBS biomolecular solvation software suite. Protein Science.

[28] Klamt, A. and Schüürmann, G. (1993). COSMO: a new approach to dielectric screening in solvents with explicit expressions for the screening energy and its gradient. Journal of the Chemical Society, Perkin Transactions 2, (5):799-805.

[29] Kuo, S. S., Altman, M. D., Bardhan, J. P., Tidor, B., and White, J. K. (2002). Fast methods for simulation of biomolecule electrostatics. In Proceedings of the 2002 IEEE/ACM International Conference on Computer-aided Design, pages 466-473. ACM.

[30] Li, L., Li, C., Sarkar, S., Zhang, J., Witham, S., Zhang, Z., Wang, L., Smith, N., Petukh, M., and Alexov, E. (2012). DelPhi: a comprehensive suite for DelPhi software and associated resources. BMC Biophysics, 5(1):9.

[31] Lipparini, F., Lagardère, L., Scalmani, G., Stamm, B., Cancès, E., Maday, Y., Piquemal, J.-P., Frisch, M. J., and Mennucci, B. (2014). Quantum calculations in solution for large to very large molecules: A new linear scaling QM/continuum approach. Journal of Physical Chemistry Letters, $5(6): 953-958$.

[32] Lipparini, F., Lagardère, L., Stamm, B., Cancès, E., Maday, Y., Piquemal, J.-P., and Mennucci, B. (2015). ddCOSMO \& ddPCM. https://www.ddpcm.org.

[33] Lipparini, F., Stamm, B., Cancès, E., Maday, Y., and Mennucci, B. (2013). Fast domain decomposition algorithm for continuum solvation models: Energy and first derivatives. Journal of Chemical Theory and Computation, 9(8):3637-3648.

[34] Liu, B., Wang, B., Zhao, R., Tong, Y., and Wei, G.-W. (2017). ESES: Software for Eulerian solvent excluded surface. Journal of Computational Chemistry, 38(7):446-466.

[35] Lu, B., Cheng, X., Huang, J., and McCammon, J. A. (2009). An Adaptive Fast Multipole Boundary Element Method for Poisson-Boltzmann Electrostatics. Journal of Chemical Theory and Computation, 5(6):1692-1699.

[36] Mallik, B., Masunov, A., and Lazaridis, T. (2002). Distance and exposure dependent effective dielectric function. Journal of Computational Chemistry, 23(11):1090-1099. 
[37] Mennucci, B. (2010). Continuum solvation models: What else can we learn from them? Journal of Physical Chemistry Letters, 1(10):1666-1674.

[38] Parter, S. V. (1999). On the Legendre-Gauss-Lobatto points and weights. Journal of Scientific Computing, 14(4):347-355.

[39] Provorse, M. R., Peev, T., Xiong, C., and Isborn, C. M. (2016). Convergence of excitation energies in mixed quantum and classical solvent: Comparison of continuum and point charge models. Journal of Physical Chemistry B, 120(47):12148-12159.

[40] Provorse Long, M. R. and Isborn, C. M. (2017). Combining explicit quantum solvent with a polarizable continuum model. Journal of Physical Chemistry B, 121(43):10105-10117.

[41] Quan, C. and Stamm, B. (2016). Mathematical analysis and calculation of molecular surfaces. Journal of Computational Physics, 322:760 - 782.

[42] Quan, C. and Stamm, B. (2017). Meshing molecular surfaces based on analytical implicit representation. Journal of Molecular Graphics and Modelling, 71:200-210.

[43] Quarteroni, A. and Valli, A. (1999). Domain decomposition methods for partial differential equations. Number CMCS-BOOK-2009-019. Oxford University Press.

[44] Raschke, T. M., Tsai, J., and Levitt, M. (2001). Quantification of the hydrophobic interaction by simulations of the aggregation of small hydrophobic solutes in water. Proceedings of the National Academy of Sciences, 98(11):5965-5969.

[45] Sauter, S. A. and Schwab, C. (2010). Boundary element methods. In Boundary Element Methods, pages 183-287. Springer.

[46] Smith, B., Bjorstad, P., and Gropp, W. (2004). Domain decomposition: parallel multilevel methods for elliptic partial differential equations. Cambridge University Press.

[47] Stamm, B., Cancès, E., Lipparini, F., and Maday, Y. (2016). A new discretization for the polarizable continuum model within the domain decomposition paradigm. Journal of Chemical Physics, 144(5):054101.

[48] Tomasi, J., Mennucci, B., and Cammi, R. (2005). Quantum mechanical continuum solvation models. Chemical Reviews, 105(8):2999-3094.

[49] Tomasi, J. and Persico, M. (1994). Molecular interactions in solution: An overview of methods based on continuous distributions of the solvent. Chemical Reviews, 94(7):2027-2094. 